\title{
Elastic behavior of Cross Laminated Timber and timber panels with regular gaps: thick-plate modeling and experimental validation
}

\author{
L. Franzoni ${ }^{1}$, A. Lebée ${ }^{1 *}$, F. Lyon ${ }^{2}$, G. Foret ${ }^{1}$ \\ ${ }^{1}$ Laboratoire Navier, UMR 8205, IFSTTAR, CNRS, UPE \\ École des Ponts ParisTech, 6 et 8 avenue Blaise Pascal \\ 77455 Marne-la-Vallée cedex-2, France \\ ${ }^{2}$ Centre Scientifique et Technique du Batiment (CSTB) \\ 84 Avenue Jean Jaurès, 77447 Marne-la-Vallée cedex-2, France \\ *e-mail: arthur.lebee@enpc.fr
}

\begin{abstract}
In the present paper, the influence of periodic gaps between lamellas of Cross Laminated Timber (CLT) on the panel's elastic behavior is analyzed by means of a periodic homogenization scheme for thick plates having periodic geometry. Both small gaps, due to the fabrication process of not-gluing lateral lamellas, and wider gaps are investigated. The results obtained with the periodic homogenization scheme are compared to existing closed-form solutions and available experimental data. It appears that the plate bending stiffness can be well predicted with both homogenization and simplified methods, while only the homogenization approach is in agreement with the experimental in-plane and out-of-plane shear behavior. The influence of several properties of CLT lay-up on the mechanical response is pointed out as well.
\end{abstract}

Keywords: Cross Laminated Timber, Timber panels with gaps, Thick plate theory, Homogenization, Engineered timber products

\section{Introduction}

Cross Laminated Timber (CLT) panels are engineered wood products with a market share in construction that has sharply increased during the last decade. They consist in lumber layers stacked and glued crosswise. Each layer has a thickness between 6 and $45 \mathrm{~mm}$ in Europe [1] and between 16 and $51 \mathrm{~mm}$ in North America [2]. The total thickness of commercial products is usually up to $300 \mathrm{~mm}$, while the span and the width can be up to $15 \mathrm{~m}$ and $3 \mathrm{~m}$. Their thick and orthogonal structure allows the application of such panels as full size walls or floors. The ease of assembly between panels and their low weight allows prefabrication, reducing construction times. The low environmental impact and high mechanical performance of CLT encourage their use in modern residential and commercial buildings instead of mineral-based materials such as concrete or masonry, as highlighted recently in a comprehensive state-of-the-art report [3].

Each layer of CLT is made of lamellas placed side by side and glued on their upper and lower faces. The lamellas may be also glued or not on their narrow edges, depending on the fabrication process. In the non-glued case, each layer features gaps between lamellas (Figure 1a). The recent European [1] and North-American [2] standard requirements for CLT allow the not-gluing of lateral lamellas (gaps allowed up to $6 \mathrm{~mm}$ in [1]). However, several experimental and numerical studies have pointed out the non-negligible influence of the gaps on the mechanical response. Indeed, dealing with the out-of-plane behavior, the studies of Hochreiner et al. [4], Flores S. et al.
[5], Franzoni et al. [6] have shown that the presence of small gaps reduces the strength of cross layers, while the influence on the global bending stiffness is low. Furthermore, the effects of small gaps are significant on CLT in-plane shear and torsional stiffness. This was shown experimentally by Brandner et al. [7] and Sebera et al. [8], who observed a reduction of the stiffness of laterally unglued CLT. In order to predict the reduced inplane shear stiffness due to unglued narrow edges, a simplified closed-form solution was developed by Moosbrugger et al. [9] and Bogensperger et al. [10]. This method is a simpler approach than a more rigorous closed-form modeling [11] but it returns a good approximation [12]. However, for gaps up to $5 \mathrm{~mm}$ such closed-form solution deviates from the experimental evidence, as pointed out by Brandner et al. [7]. The actual influence of small lateral gaps on CLT mechanical response is still to be determined and a reliable model for predicting the effects on the out-of-plane shear behavior is still missing.

Additionally, innovative lighter timber panels can be obtained when increasing the gaps between lateral lamellas up to hundreds of millimeters. The resulting space-frame like structure is a timber panel having regular voids along the two directions (Figure 1b) which can be filled by services or insulating material. Such innovative panels show therefore an improved acoustic and thermal efficiency, as well as better fire resistance, as recent industrial fire tests has established. The insulating material is less prone to falling and yields slower charring rates than with CLT. Moreover, the low volume fraction of wood within these panels allows a better exploitation of the raw material. The development of such new engineered wood prod- 


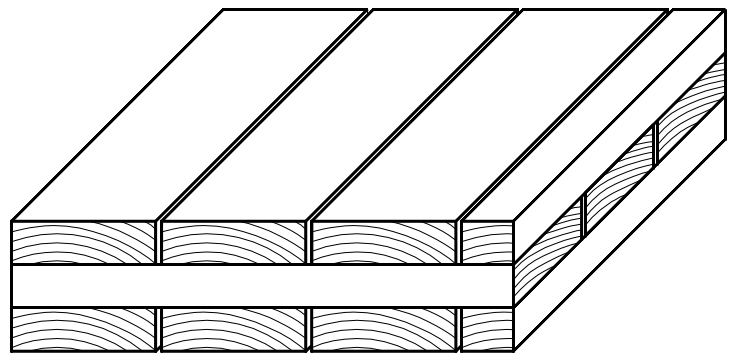

(a)

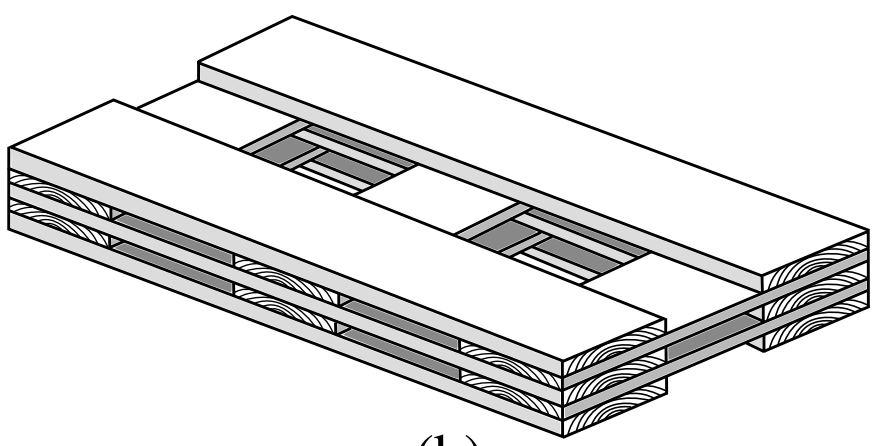

(b)

Figure 1: (a) Cross Laminated Timber panels with small gaps up to $6 \mathrm{~mm}$ as described in EN 16351 [1] and (b) innovative timber panels with large gaps

ucts is still limited because of the lack of knowledge about their mechanical behavior. Indeed currently there are no specific design methods for these products. However, several simplified design theories for standard panels exist. For instance, dealing with out-of-plane loads, the shear analogy method [13] is based on lamination theory for plates under cylindrical bending and can return a good estimate of plate bending and shear force stiffnesses. Such method can be extended to panels with large gaps reducing the mechanical properties of wood by the ratio wood/void within each layer, as Blass and Gorlacher [14] did for the shear force stiffness of timber floors with regular gaps. However, being a simplified approach, it has to be verified by means of a comparison with a more accurate modeling. Concerning in-plane shear, the closed-form solution of Moosbrugger et al. [9] derived for CLT with small gaps may be extended to larger gaps. This simplified approach has to be compared to a more refined approach and to available experimental results.

The influence of small gaps on CLT behavior has been already studied by the authors in Franzoni et al. [6]. In this first study, a discontinuous equivalent CLT layer has been defined by means of simplifying hypotheses on the not-glued layer mechanical behavior. The simplified layer behavior was combined with the exact 3D closed-form solution for laminates in bending [15] and the predicted results were in good agreement with a reference test of the literature [4]. However, the actual effects of small gaps on CLT behavior needed to be more accurately investigated, especially the in-plane behavior. Moreover, a reliable method for the structural analysis of innovative panels with large periodic gaps is still missing.

The aim of this paper is to study the influence of large and small gaps between lateral lamellas on the mechanical behavior of CLT and innovative panels by means of modeling and tests. The modeling is based on a homogenization scheme handled by a higher-order plate theory $[16,17]$. This theory for thick laminated plates, called the Bending-Gradient theory, was successfully applied to highly anisotropic laminates under cylindrical bending with various material configurations [18]. The Bending-Gradient theory was extended to plates having periodic geometry by means of a homogenization scheme, that has been successfully applied to sandwich panels [19] and beam lattices [20]. In this paper, this theory is applied to CLT panels having short or large periodic gaps within each layer. The investigated elastic behavior is related to the out-of-plane and torsional behavior of floors and to the in-plane shear behavior of diaphragms or shear walls.

The paper is organized as follows: first, in Section 2, the experimental out-of-plane and in-plane behavior of regularly voided timber panels is presented. Then, in Section 3, the BendingGradient plate theory, its related homogenization scheme and their application to the case study are summarized. Finally, Section 4 presents the comparison between the predicted and experimental behavior.

\section{Bending and in-plane shear tests of CLT and panels with regular gaps}

Few experimental studies on the mechanical behavior of CLT with gaps are present in the literature. An experimental campaign of 4-point bending tests was performed by the authors and presented in this section. Moreover, the in-plane shear tests conducted by Brandner et al. [7] are presented as well. Out-ofplane loads (involving bending and shear force stiffness) and inplane shear loads are investigated since they represent the loads involved in practice on floors and shear walls, respectively.

\subsection{Out-of-plane behavior}

The influence of gaps on the out-of-plane behavior was investigated by the authors by means of 4-point bending tests on both standard CLT and innovative panels with gaps. The panels were commercial products made from Norway spruce timber with a moisture content between $8 \%$ and $14 \%$ [2].

The standard 5-ply CLT panel had a span $L$ of $4.65 \mathrm{~m}$, a total thickness $H$ of $100 \mathrm{~mm}$ and the gaps between lateral lamellas were $s=5 \mathrm{~mm}$ on average. The lamellas of the standard CLT had a width of $w=140 \mathrm{~mm}$ and a thickness of $h=20 \mathrm{~mm}$, leading to an aspect ratio $w / h=7$.

The innovative panels with wide gaps had a span $L$ of $5.9 \mathrm{~m}$ and were made by 7 layers of equal thickness of $h=30 \mathrm{~mm}$. The width of the lamellas was $w=100 \mathrm{~mm}$, leading to an aspect ratio $w / h=3.33$. Two configurations of innovative panels (Figure 2) were tested, a configuration with gaps $s=150 \mathrm{~mm}$ and a second configuration with $s=300 \mathrm{~mm}$. Therefore they had volume fractions of respectively $\lambda=0.4$ and $\lambda=0.25$, where $\lambda=w / w+s$. Figure 2 and Table 1 present respectively a part of typical cross section of timber panel with gaps and the geometric properties of the tested panels. 


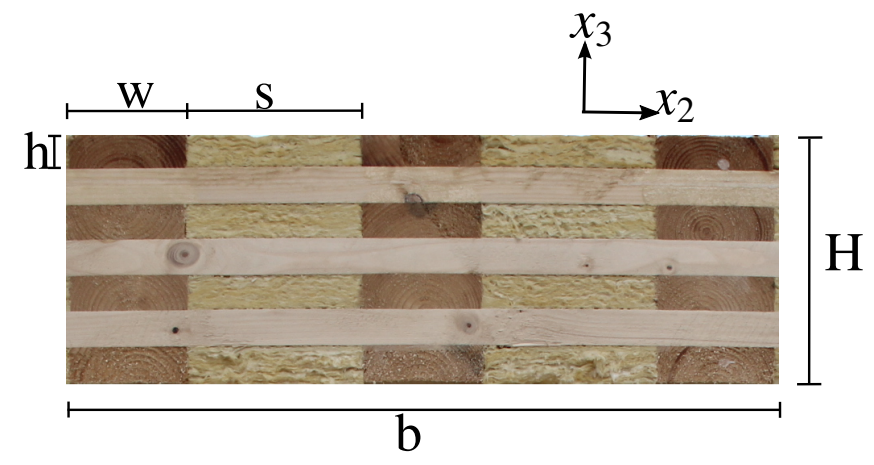

Figure 2: Part of a typical cross section of an innovative timber panel with regular gaps $(s=150 \mathrm{~mm}, w=100 \mathrm{~mm}, h=30 \mathrm{~mm})$

\begin{tabular}{lccc}
\hline & $\mathrm{s}=5 \mathrm{~mm}$ & $\mathrm{~s}=150 \mathrm{~mm}$ & $\mathrm{~s}=300 \mathrm{~mm}$ \\
\hline $\mathrm{L}[\mathrm{m}]$ & 4.65 & 5.9 & 5.9 \\
$\mathrm{~b}[\mathrm{~m}]$ & 1.25 & 1.31 & 1.26 \\
$\mathrm{H}[\mathrm{mm}]$ & 100 & 210 & 210 \\
layers & 5 & 7 & 7 \\
$\mathrm{~h}[\mathrm{~mm}]$ & 20 & 30 & 30 \\
$\mathrm{w}[\mathrm{mm}]$ & 140 & 100 & 100 \\
$\lambda$ & 0.95 & 0.4 & 0.25 \\
\hline
\end{tabular}

Table 1: Properties of the tested panels

The voids of specimens with large gaps were filled with insulating material (glass wool) having negligible mechanical properties compared to wood. The specimens were simply supported on two sides and submitted to a symmetric 4-point bending. The measurement system was based on vertical LVDTs to measure the panel's curvature. Figure 3 shows the 4-point bending set-up.

The panel's bending stiffness has been derived from the measured curvature between the loading devices. Then, knowing the bending stiffness and the global mid-span deflection, the shear force stiffness and the ratio between shear deflection and bending deflection $\alpha=U_{\text {shear }} / U_{\text {bending }}$ can be also obtained (for
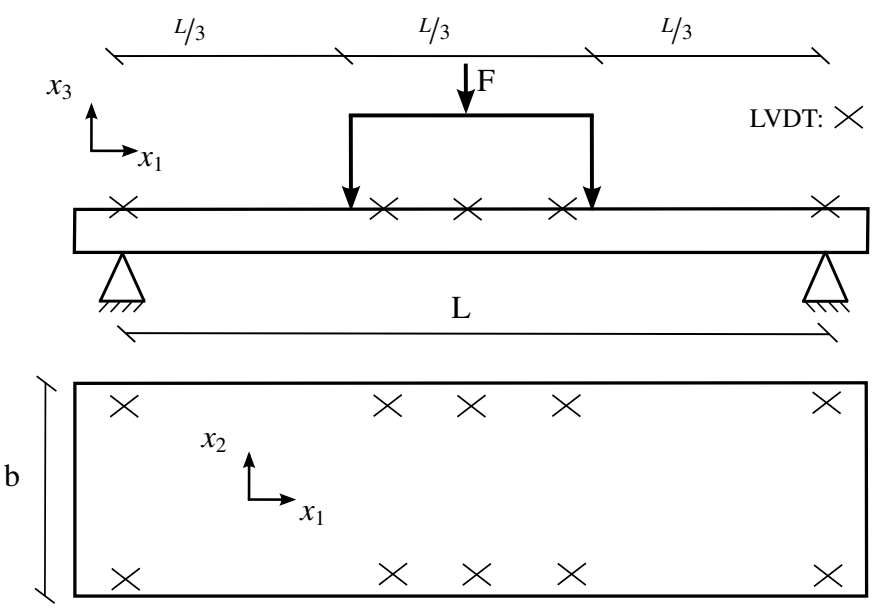

Figure 3: 4-point bending test set-up and position of LVDTs

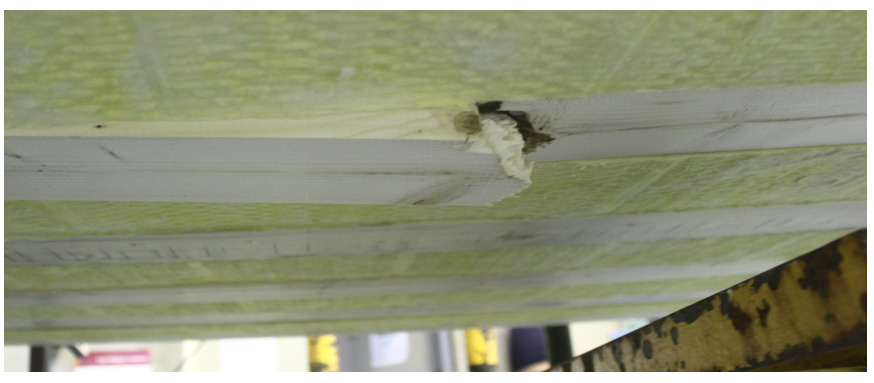

(a)

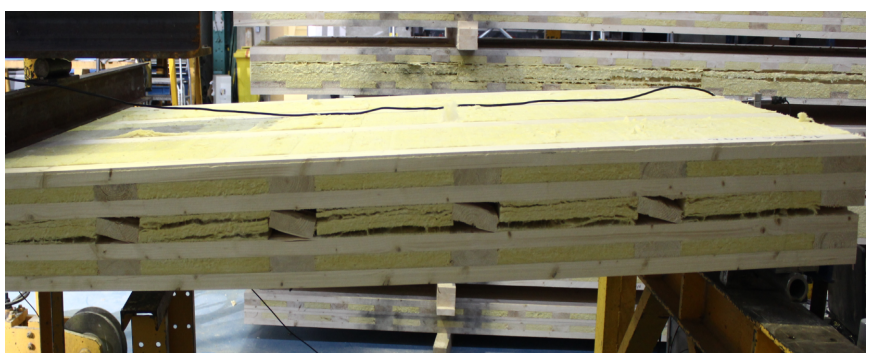

(b)

Figure 4: Failure modes of panels with regular gaps: (a) longitudinal tensile failure on bottom layer of $\mathrm{s}=150 \mathrm{~mm}$ panel and (b) shear failure of cross lamellas of $\mathrm{s}=300 \mathrm{~mm}$ panel

more details see Franzoni et al. [28]).

A first cycle of load/unload up to $20 \%$ of the expected failure load was performed, with a following increase of load until failure of the panel. Two specimens for each configuration shown in Table 1 were tested. Table 2 presents the experimental results in terms of failure load, failure mode, bending $\left(b \cdot D_{11}\right)$ and shear force stiffness $\left(b \cdot F_{11}\right)$. As detailed in Section 2, $D_{11}$ is the plate bending stiffness in direction $x_{1}$ and $b \cdot D_{11}$ is thus the corresponding beam bending $(E I)$ stiffness. Similarly, $F_{11}$ is the thick plate shear force stiffness and $b \cdot F_{11}$ the corresponding beam shear $(G A)$ stiffness. Note that, due to the heterogeneity of the panel, the stiffness moduli $(E, G)$ may not be directly separated from the geometric quantities $(I, A)$ which motivates this specific notation. The failure mode denoted RS stands for the rolling shear failure due to shear stress in the wood RadialTangential plane which yields a rotation of wood's fibers (see for instance $[14,6,25])$.

Table 2 shows that when enlarging the gaps within CLT layers, the failure load and the panel's stiffness decreases. At the same time, enlarging the gaps leads to increasing shear compliance, increasing the shear contribution to the deflection $\alpha$ (Table 2) and changing the failure mode. Indeed, while the standard CLT and the $s=150 \mathrm{~mm}$ panel failed in traction in the bottom layers due to bending stresses (Figure 4a), the most spaced configuration with $s=300 \mathrm{~mm}$ failed in rolling shear with a rotation of cross lamellas (Figure $4 b$ ).

\subsubsection{Small-scale tests}

The raw timber of the panels (Norway spruce) has been subsequently tested in tension, compression and shear in order to determine the wood elastic moduli. Standard and in- 


\begin{tabular}{lcccccc}
\hline & $s=5 \mathrm{~mm}$ & $s=5 \mathrm{~mm}$ & $s=150 \mathrm{~mm}$ & $s=10 \mathrm{~mm}$ & $s=300 \mathrm{~mm}$ & $s=300 \mathrm{~mm}$ \\
& $(1)$ & $(2)$ & $(1)$ & $(2)$ & $(1)$ & $(2)$ \\
\hline$F_{\max }[\mathrm{kN}]$ & 77 & 82 & 72 & 65 & 34 & 33 \\
Failure mode & $\mathrm{TL}$ & $\mathrm{TL}$ & $\mathrm{TL}$ & $\mathrm{TL}$ & $\mathrm{RS}$ & $\mathrm{RS}$ \\
Global stiffness $[\mathrm{kN} \cdot \mathrm{mm}]$ & 485 & 490 & 850 & 810 & 430 & 390 \\
$b \cdot D_{11}\left[\mathrm{kN} \cdot \mathrm{m}^{2}\right]$ & 890 & 900 & 3600 & 3340 & 2025 & 1790 \\
$b \cdot F_{11}[\mathrm{kN}]$ & 12300 & 12700 & 5800 & 5700 & 1850 & 1790 \\
$\alpha=U_{\text {shear }} / U_{\text {bending }}$ & 0.03 & 0.03 & 0.17 & 0.16 & 0.29 & 0.27 \\
\hline
\end{tabular}

Table 2: Main results of 4-point bending tests. The gaps $s$ are in $\mathrm{mm} . \mathrm{TL}=$ tensile failure in longitudinal direction of bottom layer; $\mathrm{RS}=$ rolling-shear failure in middle layer

novative panels were supplied by two different producers and the respective raw woods were graded following two different systems. According to the producers' declaration, the standard panels were made up of S10 timber following the German visual classification [22], while the timber of innovative panels were certified as C24 following EN 338 [23].

Wood is an orthotropic material with three principal axes: the first one is aligned with the fiber or trunk direction (longitudinal direction $\mathrm{L}$ ) while in the transverse plane, the remaining two axes are orthogonal to the annual rings (radial direction, R) and tangential (tangential direction, T) (Figure 5). However, the local variation of the annual rings within CLT lamellas as well as the related actual position of orthotropic coordinates are unknown. Hence, a lamella's reference frame $(L, N, Z)$ instead of orthotropic coordinates $(L, T, R)$ (Figure 5$)$ is considered in this study. The radial and tangential coordinates are therefore mixed into the two generalized transverse directions $N$ and $Z$, independently from the annual rings pattern.

The small-scale tests were performed with respect to $(L, N, Z)$ reference frame and Table 3 shows the obtained elastic properties, in agreement with several studies in the literature [24, 25 , 26]. Due to a lack of material, it was possible to determine only the rolling-shear $G_{Z N}$ modulus of standard CLT and not the $G_{L Z}$ shear modulus. Moreover, the thinness of the lamellas prevented shear tests in the $L N$ plane, hence the wood shear modulus $G_{L N}$ is considered to equal shear $G_{L Z}$ modulus in the modeling. More details about the small-scale tests on the raw timber of panels can be found in [27] and [28].

\subsection{In-plane shear behavior}

The in-plane shear behavior of CLT has been the object of a recent experimental campaign performed by Brandner et al. [7]. The authors tested portions of CLT panels rotated $45^{\circ}$ and submitted to a uni-axial compression in order to obtain a stress state close to pure in-plane shear in the CLT conventional reference frame. The aim of this experimental work was to develop a new testing configuration and to study the influence on inplane shear behavior of several parameters such as the lay-up, the width of the lamella and the manufacturing of the gap. The latter turned out to be the most influential parameter on CLT inplane shear behavior. Indeed, the reduction of the in-plane shear stiffness when passing from glued to unglued lateral lamellas was about $30 \%$ and even $50 \%$ for a $5 \mathrm{~mm}$ gap. Table 4 shows the properties of the so-called "series A" tested by Brandner et al. [7] and the obtained in-plane shear stiffnesses.

\section{Modeling}

In this section, we first briefly introduce the Bending-Gradient plate theory. Full details about this theory can be found in [16, 17, 18]. Then, the application of the plate theory to a periodic geometry is presented, with focus on CLT and panels with regular gaps.

\subsection{Summary of the Bending-Gradient model}

CLT with small gaps as well as aerated CLT may be considered as in-plane periodic structures since they consist of a repetitive pattern. Finite element modeling of such structures requires a very fine mesh. Hence, in order to spare computational burden, it is convenient to seek an equivalent membrane and plate model for these 3D structures by means of a homogenization scheme. Additionally, according to the significant shear effects found during the experimental investigations (see Table 2), knowing the deflection related to transverse shear effects is also necessary for engineering applications of CLT and innovative products with gaps. This suggests applying the Bending-Gradient theory which is an extension of the well known Reissner-Mindlin thick plate theory to the case of heterogeneous plates.

Let us recall that the usual generalized stresses for the ReissnerMindlin plate are the membrane stress $N_{\alpha \beta}$, the bending moment $M_{\alpha \beta}$, and the shear force $Q_{\alpha}$. These generalized stresses are respectively dual with the membrane strain $e_{\alpha \beta}$, the curvature $\chi_{\alpha \beta}$, and the transverse shear distortion $\gamma_{\alpha}$. Assuming the plate is symmetric with respect to its mid-plane, these variables are related through the following constitutive equations:

$$
\left\{\begin{array}{l}
N=A: e, \\
M=D: \chi, \\
Q=F \cdot \gamma,
\end{array}\right.
$$

where $\boldsymbol{A}$ is the membrane fourth order stiffness tensor, $\boldsymbol{D}$ is the bending fourth order stiffness tensor and $\boldsymbol{F}$ is the shear force second order stiffness tensor. The simple, double and triple contraction products are hereinafter defined as follows: $\boldsymbol{X} \cdot \boldsymbol{Y}=X_{\alpha} Y_{\alpha}, \boldsymbol{X}: \boldsymbol{Y}=X_{\alpha \beta} Y_{\beta \alpha}$ and $\boldsymbol{X}: \boldsymbol{Y}=X_{\alpha \beta \gamma} Y_{\gamma \beta \alpha}$, with Greek letters that stand for the in-plane coordinates $\left(x_{1}, x_{2}\right)$. 

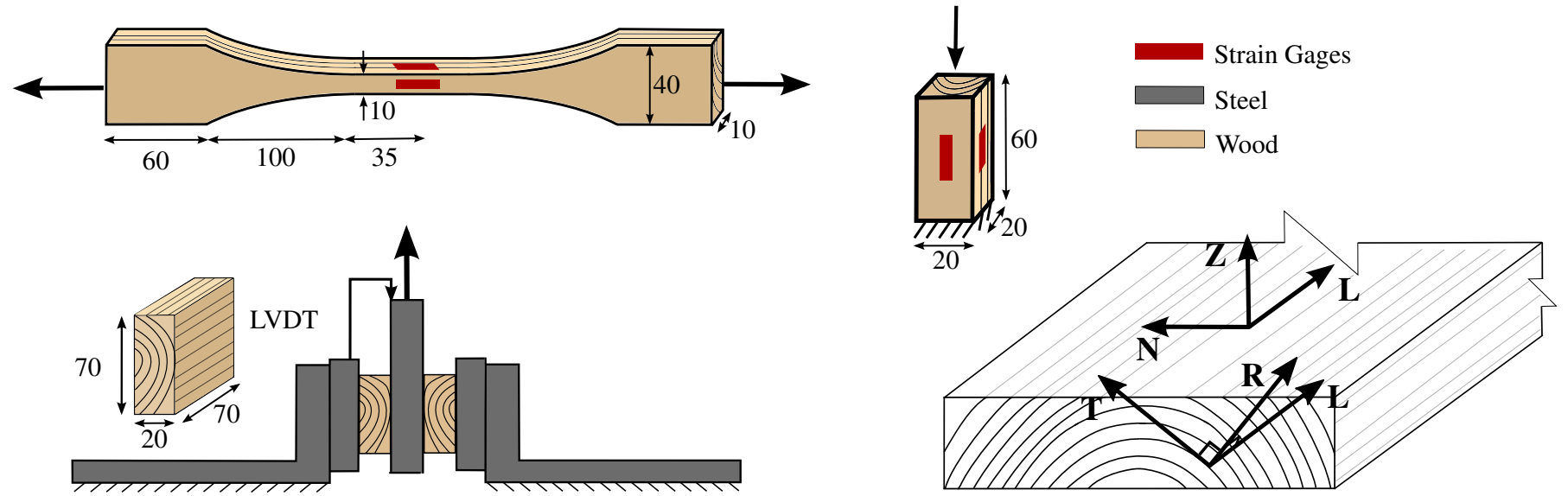

Figure 5: Geometry of the small-scale timber specimens (dimensions in $\mathrm{mm}$ ) and lamella's reference frame $(L, N, Z)$ considered instead of wood's orthotropic coordinates $(L, T, R)$

\begin{tabular}{lcccccccc}
\hline & $E_{L}$ & $E_{N}$ & $E_{Z}$ & $G_{Z N}$ & $G_{L Z}$ & $v_{Z N}$ & $v_{L Z}$ & $v_{L N}$ \\
\hline Innovative panels & & & & & & & & \\
\hline number of samples & 21 & 6 & 5 & 10 & 6 & 5 & 8 & 8 \\
Mean [MPa]/[1] & 12500 & 530 & 400 & 110 & 580 & 0.71 & 0.35 & 0.51 \\
COV [\%] & 16 & 25 & 7 & 27 & 14 & 6 & 16 & 12 \\
\hline Standard panels & & & & & & & & \\
\hline number of samples & 17 & 6 & 5 & 8 & - & 5 & 8 & 8 \\
Mean [MPa]/[1] & 10000 & 620 & 440 & 75 & - & 0.69 & 0.38 & 0.49 \\
COV [\%] & 11 & 28 & 6 & 25 & - & 10 & 14 & 6 \\
\hline
\end{tabular}

Table 3: Obtained elastic properties of the raw wood. Poisson's ratios $v$ are unit-less 


\begin{tabular}{lccc}
\hline & A1 & A2 & A3 \\
\hline $\mathrm{h}[\mathrm{mm}]$ & 30 & 30 & 30 \\
$\mathrm{w}[\mathrm{mm}]$ & 160 & 160 & 160 \\
layers & 3 & 3 & 3 \\
$\mathrm{~s}[\mathrm{~mm}]$ & glued & 0 & 5 \\
In-plane shear modulus [MPa] & 650 & 475 & 310 \\
\hline
\end{tabular}

Table 4: Properties of the tested specimen and measured in-plane shear stiffness by Brandner et al. [7]

Whereas the derivation of $\boldsymbol{A}$ and $\boldsymbol{D}$ is well established in the literature for laminated plates (Classical Lamination Theory) as well as for periodic plates [29, 30]; the derivation of $\boldsymbol{F}$ raised many difficulties. Indeed, when the plate is heterogeneous, additional variables are required to describe accurately transverse shear deformations.

In the Bending-Gradient theory, the conventional shear force $\boldsymbol{Q}$ with two degrees of freedom is replaced by the generalized shear force $\boldsymbol{R}$ with six degrees of freedom. The generalized shear force is a third order tensor. With this new set of variables, the constitutive equation becomes:

$$
\left\{\begin{array}{l}
N=A: e \\
M=D: \chi \\
R=H: \Gamma
\end{array}\right.
$$

where $\boldsymbol{\Gamma}$ is the generalized shear distortion and $\boldsymbol{H}$ the generalized shear force sixth-order stiffness tensor. In case $\boldsymbol{H}$ is not invertible, $\boldsymbol{R}$ and $\boldsymbol{\Gamma}$ are restricted to a specific vector space detailed in $[31,32]$. These equations may be written in terms of compliance as follows:

$$
\left\{\begin{array}{l}
e=a: N \\
\chi=d: M \\
\Gamma=h: R
\end{array}\right.
$$

where $\boldsymbol{a}, \boldsymbol{d}$ and $\boldsymbol{h}$ are respectively the membrane, bending and generalized shear force compliance tensors (reciprocals of $\boldsymbol{A}$, $\boldsymbol{D}$ and $\boldsymbol{H}$ ).

Depending on the plate micro-structure, the Bending-Gradient theory may be turned into the Reissner-Mindlin plate theory. For instance, this is the case when the plate is homogeneous. In [16], the relative distance between both plate theories was introduced as $0 \leq \Delta^{\mathrm{RM} / \mathrm{BG}}<1$. When $\Delta^{\mathrm{RM} / \mathrm{BG}}=0$, the BendingGradient theory exactly reduces to a Reissner-Mindlin theory and we have the following direct estimation of Reissner-Mindlin shear force stiffness moduli as function of the Bending-Gradient ones for an orthotropic plate:

$$
\frac{1}{h_{11111}}=F_{11} \text { and } \quad \frac{1}{h_{22222}}=F_{22} \text {. }
$$

When dealing with laminated plates it is possible to derive $\boldsymbol{A}, \boldsymbol{D}$ and $\boldsymbol{h}$ directly from the constitutive material behavior. However, with periodic plates this requires a homogenization procedure which is detailed in the next section.

\subsection{Homogenization scheme}

Let us consider a plate generated by periodicity of a unitcell $Y$ according to the in-plane Directions $x_{1}$ and $x_{2}$. The upper face $\partial Y_{3}^{+}$and the lower face $\partial Y_{3}^{-}$are traction free and the lateral faces $\partial Y_{l}$ connect adjacent unit-cells. $A_{Y}$ is the area of the unitcell cross section with the plate mid-plane. $\boldsymbol{x}=\left(x_{1}, x_{2}, x_{3}\right)$ is the set of coordinates in the unit-cell reference frame.

Finding $\boldsymbol{A}, \boldsymbol{D}$ and $\boldsymbol{h}$ requires the resolution of unit-cell problems. The membrane $\boldsymbol{A}$ and bending $\boldsymbol{D}$ stiffness tensors are derived by means of a first unit-cell problem which also gives the corresponding stress states. Then solving a second unit-cell problem is necessary for deriving $\boldsymbol{h}$.

\subsubsection{Membrane and thin plate unit-cell problem}

Homogenization of periodic plates at leading order was first established by Caillerie [29]. The unit-cell problem is stated as follows:

$$
\mathcal{P}^{(e, \chi)}\left\{\begin{array}{l}
\operatorname{div} \boldsymbol{\sigma}^{(e, \chi)}=0, \\
\boldsymbol{\sigma}^{(e, \chi)}=\boldsymbol{C}(\boldsymbol{x}): \boldsymbol{\varepsilon}^{(e, \chi)}, \\
\boldsymbol{\varepsilon}^{(e, \chi)}=\hat{\boldsymbol{e}}+x_{3} \hat{\chi}+\operatorname{grad}^{s} \boldsymbol{u}^{\mathrm{per}}, \\
\boldsymbol{\sigma} \cdot \boldsymbol{e}_{3}=0 \text { on free faces } \partial Y_{3}^{ \pm}, \\
\boldsymbol{\sigma} \cdot \boldsymbol{n} \text { skew-periodic on } \partial Y_{l}, \\
\boldsymbol{u}^{\mathrm{per}}\left(x_{1}, x_{2}, x_{3}\right) \quad\left(x_{1}, x_{2}\right) \text {-periodic on } \partial Y_{l},
\end{array}\right.
$$

where $\boldsymbol{u}^{\text {per }}$ is the $3 \mathrm{D}$ displacement vector, $\boldsymbol{\varepsilon}$ is the linearized strain second-order tensor, $\sigma$ is the Cauchy stress second-order tensor, $\boldsymbol{C}$ is the 3D stiffness fourth-order tensor. Furthermore, $\operatorname{grad}^{s}$ is the symmetric part of the 3D gradient operator, div is the 3D divergence and $\boldsymbol{n}$ is the outer normal to the boundary of the unit-cell.

This problem enforces the membrane strains $\boldsymbol{e}$ and the curvatures $\chi$ on average on the unit-cell while taking into account periodicity in the $\left(x_{1}, x_{2}\right)$-plane and traction-free conditions on the upper and lower faces of the plate. In Equation (5), $\hat{\boldsymbol{e}}$ and $\hat{\boldsymbol{\chi}}$ denote the out-of-plane extension of the in-plane tensors $\boldsymbol{e}$ and $\chi$ :

$$
\boldsymbol{e}=\left(\begin{array}{ccc}
e_{11} & e_{12} & 0 \\
e_{21} & e_{22} & 0 \\
0 & 0 & 0
\end{array}\right) \text { and } \chi=\left(\begin{array}{ccc}
\chi_{11} & \chi_{12} & 0 \\
\chi_{21} & \chi_{22} & 0 \\
0 & 0 & 0
\end{array}\right)
$$

Solving the problem for each individual component of $\boldsymbol{e}$ and $\boldsymbol{\chi}$ leads to the localization stress fields $s^{(e)}$ and $s^{(\chi)}$ such that the total stress $\sigma^{(e, \chi)}$ solution of the problem $\mathcal{P}^{(e, \chi)}$ is recovered by linear combinations:

$$
\sigma_{i j}^{(e, \chi)}=s_{i j \alpha \beta}^{(e)}(\boldsymbol{x}) e_{\beta \alpha}+s_{i j \alpha \beta}^{(\chi)}(\boldsymbol{x}) \chi_{\beta \alpha} .
$$

Hence, $\sigma^{(e, \chi)}$ is the stress state inside any unit-cell when the periodic plate is subjected to given uniform membrane strain and curvature.

The membrane and thin plate stiffness tensors are then evaluated as follows:

$$
\boldsymbol{A}=\left\langle{ }^{T} \boldsymbol{s}^{(e)}: \boldsymbol{C}^{-1}: \boldsymbol{s}^{(e)}\right\rangle, \quad \boldsymbol{D}=\left\langle{ }^{T} \boldsymbol{s}^{(\chi)}: \boldsymbol{C}^{-1}: \boldsymbol{s}^{(\chi)}\right\rangle
$$


where:

$$
\langle f\rangle=\frac{1}{A_{Y}} \int_{Y} f(\boldsymbol{x}) d Y
$$

is the normalized average (surface average) on the unit-cell and the superscript ${ }^{T}$ stands for the transpose operator. Finally, using the inverted plate constitutive law of Equation (1a) and Equation (1b) and localization tensors $\boldsymbol{s}^{(e)}$ and $\boldsymbol{s}^{(\chi)}$, it is possible to write the local stress field generated by the membrane stress and the bending moment as:

$$
\begin{gathered}
\sigma^{(N)}=s^{(N)}: \boldsymbol{N}=\left(\boldsymbol{s}^{(e)}: \boldsymbol{a}\right): \boldsymbol{N}, \\
\boldsymbol{\sigma}^{(M)}=\boldsymbol{s}^{(M)}: \boldsymbol{M}=\left(\boldsymbol{s}^{(\chi)}: \boldsymbol{d}\right): \boldsymbol{M} .
\end{gathered}
$$

\subsubsection{The generalized shear force unit-cell problem}

The generalized shear auxiliary problem on the unit-cell is defined as:

$$
\mathcal{P}^{(R)}\left\{\begin{array}{l}
\operatorname{div} \sigma^{(R)}+f^{(R)}(\boldsymbol{x})=0 \\
\sigma^{(R)}=\boldsymbol{C}(\boldsymbol{x}):\left(\operatorname{grad}^{S} \boldsymbol{u}^{(R)}\right), \\
\boldsymbol{\sigma}^{(R)} \cdot \boldsymbol{e}_{3}=0 \text { on free faces } \partial Y_{3}^{ \pm} \\
\boldsymbol{\sigma}^{(R)} \cdot \boldsymbol{n} \text { skew-periodic on } \partial Y_{l}, \\
\boldsymbol{u}^{(R)}\left(x_{1}, x_{2}, x_{3}\right) \quad\left(x_{1}, x_{2}\right) \text {-periodic on } \partial Y_{l},
\end{array}\right.
$$

where the load related to the generalized shear force is written as:

$$
f_{i}^{(R)}=s_{i \alpha \beta \gamma}^{(M)}(x) R_{\gamma \beta \alpha} .
$$

Solving $\mathcal{P}^{(R)}$ for each component of $\boldsymbol{R}$ leads to the localization stress field $s_{i j \alpha \beta \gamma}^{(R)}$ associated with $\boldsymbol{R}$. The overall stress is obtained by the linear combination:

$$
\sigma_{i j}^{(R)}=s_{i j \alpha \beta \gamma}^{(R)}(\boldsymbol{y}) R_{\gamma \beta \alpha} .
$$

It is then possible to identify the Bending-Gradient compliance tensor as:

$$
\boldsymbol{h}=\left\langle\left(\boldsymbol{s}^{(R)}\right): \boldsymbol{C}^{-1}(\boldsymbol{x}): \boldsymbol{s}^{(R)}\right\rangle .
$$

\subsection{Application to CLT and timber panels with regular gaps}

A unit-cell of a 5-ply timber panel with gaps is shown in Figure 6. The lateral faces are periodic along the $x_{1}$ and $x_{2}$ directions, the upper and lower faces are free and the layers are rigidly connected. The investigated lay-ups in this study are 3, 5 and 7-ply panels. Moreover, the studies of Moosbrugger et al. [9] and Silly [33] highlighted the great influence of the lamella aspect-ratio width to thickness $w / h$. Therefore two different lamella aspect-ratios $w / h$ are also analyzed: $w / h=3.33$ and $w / h=10$. This is achieved by fixing the width at $100 \mathrm{~mm}$, while the considered thicknesses are 30 or $10 \mathrm{~mm}$ (Figure 6) which is within the standard range from 6 to $45 \mathrm{~mm}$ for CLT layers' thickness established in EN 16351 [1].

The auxiliary problems are solved by means of a finite elements software in order to obtain the elastic strain energy of the unit-cell. Solid C3D8 linear elements in ABAQUS are chosen [34]. When the gaps are very narrow, the size of an element should be smaller than the gap width. A convergence study, not detailed here, led to a mesh of minimum twelve elements per gap width, as shown in the detail of Figure 7, and of minimum six elements per layer's thickness. This is in order to limit the stiffness relative error to $1 \%$. The timber elastic engineering constants for the FEM modeling are taken from Table 3.

In this paper, the unit-cell of the considered panels features three planes of symmetry. It is possible to study only one eighth of the unit-cell as shown in Figure 6, reducing the computational costs.

Furthermore, these planes of symmetry allow simple symmetric or skew-symmetric boundary conditions on lateral faces, instead of the periodic boundary conditions required in the general case. The load of the thin-plate and membrane homogenization are both imposed membrane $\boldsymbol{e}$ and curvature $\chi$ displacements on the boundaries and are detailed in Figure 8 depending on the considered unit load.

Similarly, the generalized shear force unit-cell problem is parametrized by the six components of $\boldsymbol{R}$ and the corresponding boundary conditions are detailed in Figure 9.

The symmetries of the investigated panels lead also to simplifications of the constitutive equations. When the unit-cell is unchanged through a $\pi$ angle rotation with respect to a vertical axis, then the membrane and thin-plate stresses $(\boldsymbol{N}, \boldsymbol{M})$ are uncoupled from $\boldsymbol{R}$. When the unit-cell follows the mirror symmetry with respect to the mid-plane, membrane stress are uncoupled from bending moments. Finally, the plate is orthotropic when the unit-cell is invariant through a vertical plane symmetry. When all these symmetries occurs, there remain 4 moduli for membrane stress, 4 moduli for bending moments and 12 moduli for the bending gradient [18] and the plate moduli $\boldsymbol{A}, \boldsymbol{D}$ and $\boldsymbol{h}$ have the following form, in Kelvin notation:

$$
\begin{aligned}
& \boldsymbol{A}=\left(\begin{array}{ccc}
A_{1111} & A_{1122} & 0 \\
& A_{2222} & 0 \\
\text { sym } & & 2 A_{1212}
\end{array}\right)=\left(\begin{array}{ccc}
A_{11} & A_{12} & 0 \\
& A_{22} & 0 \\
\text { sym } & & A_{33}
\end{array}\right), \\
& \boldsymbol{D}=\left(\begin{array}{ccc}
D_{1111} & D_{1122} & 0 \\
& D_{2222} & 0 \\
\text { sym } & & 2 D_{1212}
\end{array}\right)=\left(\begin{array}{ccc}
D_{11} & D_{12} & 0 \\
& D_{22} & 0 \\
\text { sym } & & D_{33}
\end{array}\right) \text {, } \\
& \boldsymbol{h}=\left(\begin{array}{cccccc}
h_{111111} & h_{111112} & 0 & 0 & 0 & \sqrt{2} h_{111221} \\
& h_{221122} & 0 & 0 & 0 & h_{221221} \\
& & 2 h_{121121} & \sqrt{2} h_{121211} & \sqrt{2} h_{121222} & 0 \\
& & & h_{112211} & h_{112222} & 0 \\
& \text { sym } & & & h_{2222222} & 0 \\
& & & & & 2 h_{122221}
\end{array}\right) \\
& =\left(\begin{array}{cccccc}
h_{11} & h_{12} & 0 & 0 & 0 & h_{16} \\
& h_{22} & 0 & 0 & 0 & h_{26} \\
& & h_{33} & h_{34} & h_{35} & 0 \\
& & & h_{44} & h_{45} & 0 \\
& s y m & & & h_{55} & 0 \\
& & & & & h_{66}
\end{array}\right) .
\end{aligned}
$$

\section{Results}

In this section, results from the plate homogenization procedure are presented and compared to simplified approaches as well as to available experimental data. First, the variation of plate moduli is plotted as a function of the size of gaps $s$ within 

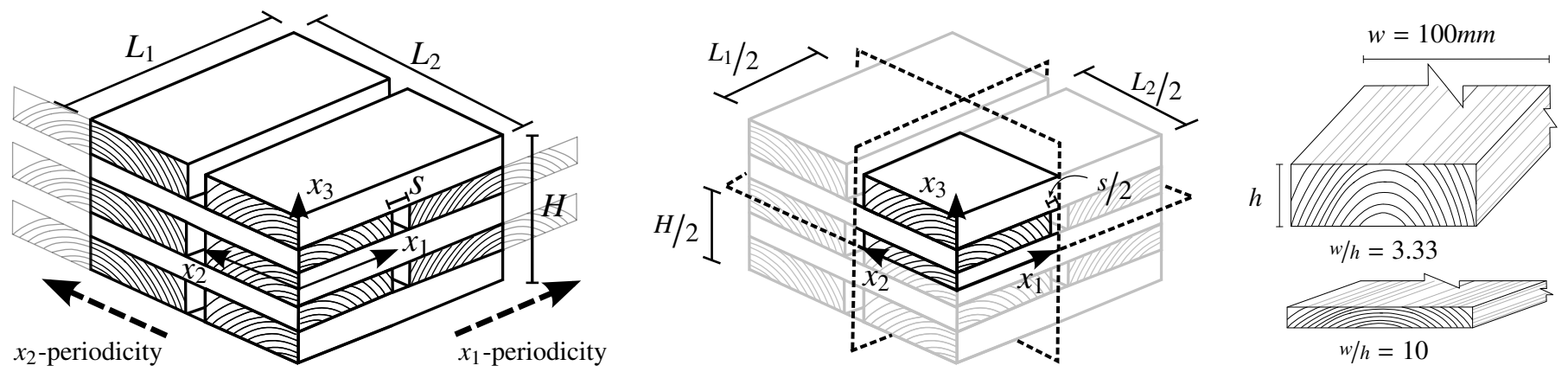

Figure 6: Complete (left) and one-eighth (middle) unit-cell of 5-ply timber panel with gaps and investigated ratios $w / h$ of lamellas (right)

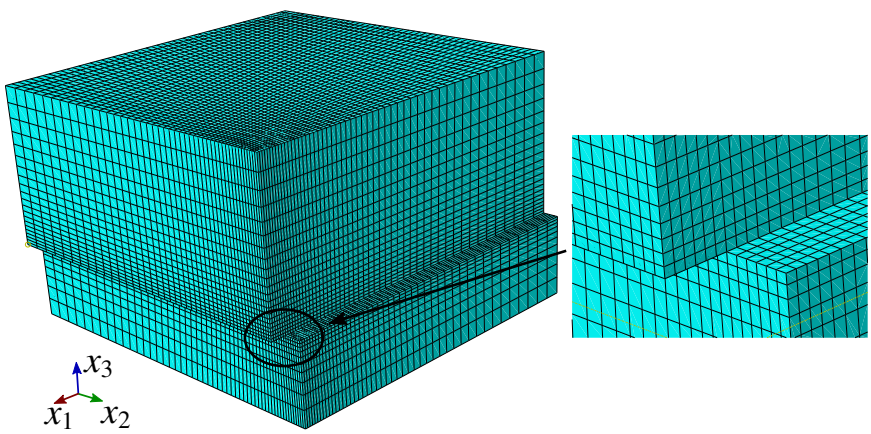

Figure 7: FE mesh of one eighth 3-ply unit-cell with zoom on half a gap (5 mm) mesh

the panel. Experimental or computed values of a plate stiffness $K$ are normalized with the corresponding stiffness $K^{*}$ of a continuous panel having the narrow edges glued, derived from the plate homogenization scheme. Finally, the predicted variation of the stress inside the panel as a function of gaps is compared to the experimental results of 4-point bending tests.

\subsection{Bending stiffness}

The measured bending stiffness of the tested panels is now compared to the stiffness predicted with the plate homogenization procedure $\left(D_{11}\right)$ and with the Classical Lamination Theory combined with the reduced properties of wood by the volume fraction coefficient $\lambda$. The normalized bending stiffness of CLT and innovative panels is plotted in Figure 10, where a very good agreement is found both with homogenization and closed-form approaches. Similar results were found for the membrane stiffnesses $A_{11}$ and $A_{22}$ and also for the bending stiffness $D_{22}$. The comparison was also made for 3-,5 and 7-ply and several aspect ratios with similar observations.

It is not surprising that the Classical Lamination Theory weighed with the volume fraction already yields good result since either glued or not, cross lamellas have a very small contribution to the bending stiffness. The homogenization procedure confirms this observation. A very small influence of free narrow edges is to be noted for small gaps in Figure 10 where the homogenized stiffness slightly deviates from the closedform approach. (a) Symmetric loadings $e_{11}, e_{22}, \chi_{11}, \chi_{22}$

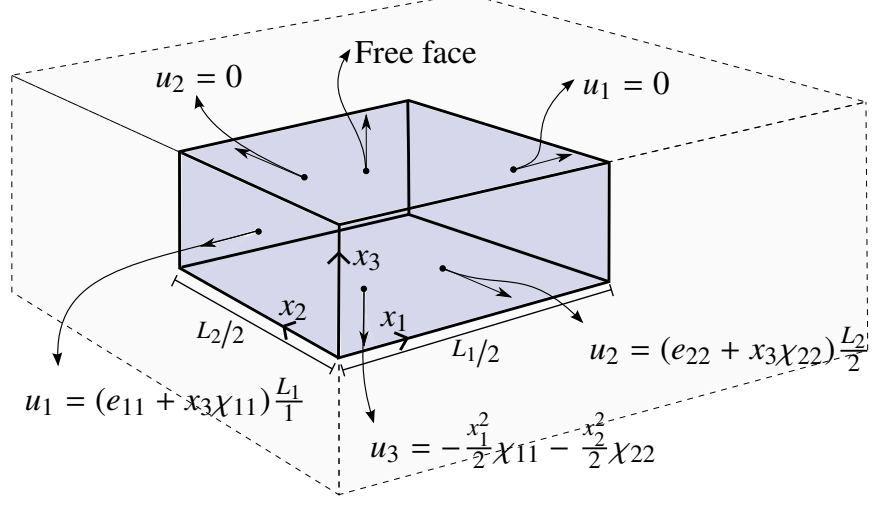

(b) Skew-symmetric loadings $e_{12}, \chi_{12}$

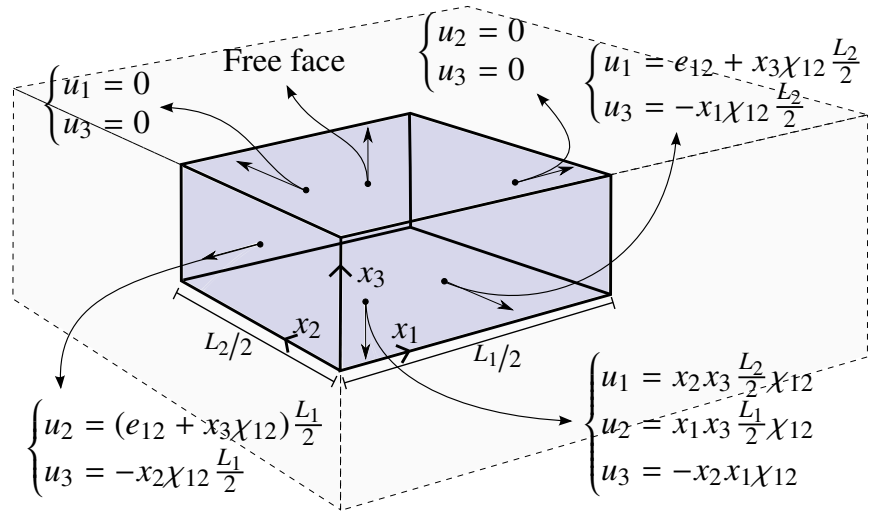

Figure 8: Applied boundary conditions on $1 / 8$ of the unit-cell for the bending and membrane loadings. Symmetric (a) and skew-symmetric (b) loadings. 


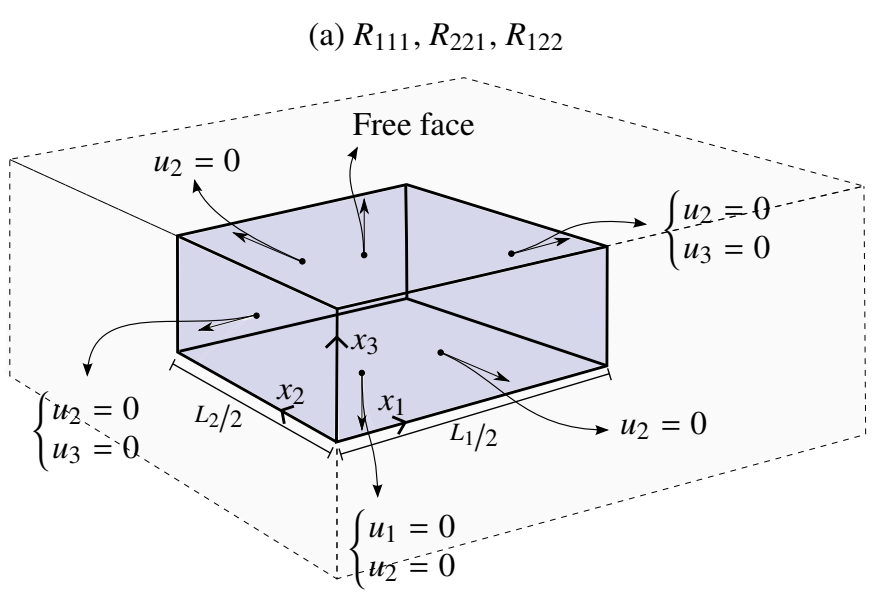

(b) $R_{121}, R_{112}, R_{222}$

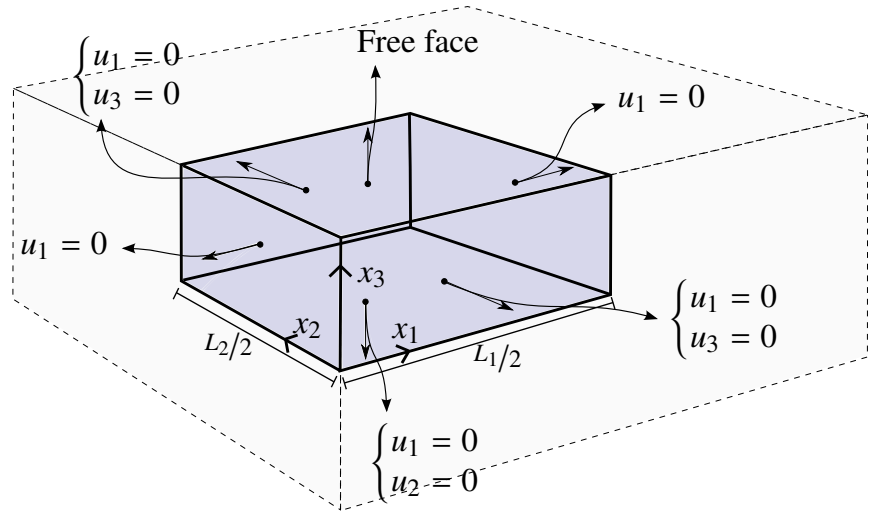

Figure 9: Applied boundary conditions on $1 / 8$ of the unit-cell for the generalized shear loadings: $R_{111}, R_{221}, R_{122}$ loadings (a) and $R_{121}, R_{112}, R_{222}$ loadings (b)

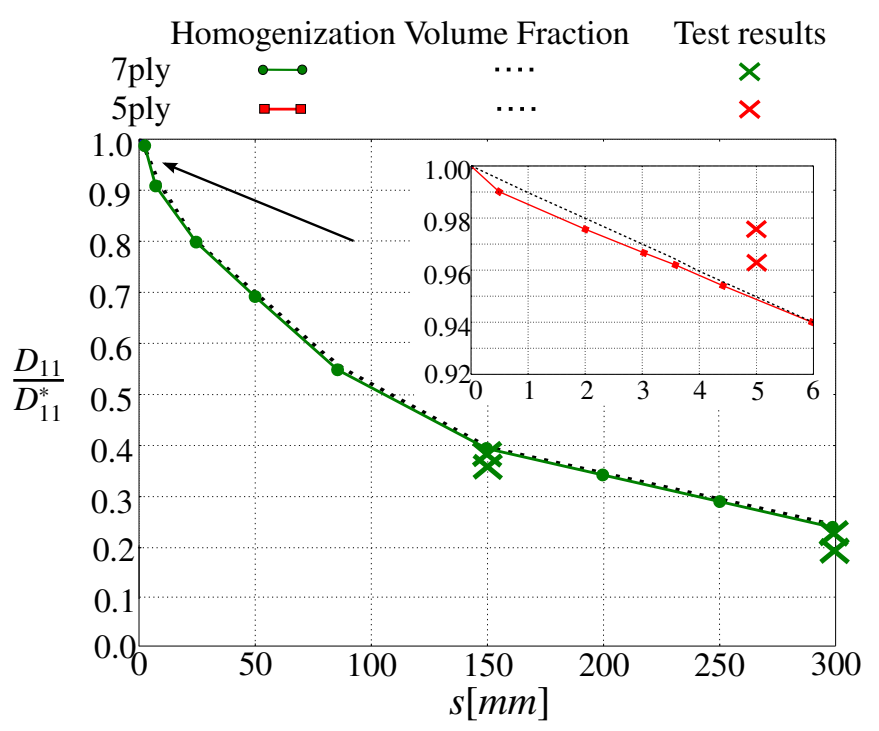

Figure 10: Comparison between the experimental and predicted bending stiffness $D_{11}$ of timber panels with gaps

\subsection{In-plane shear and torsional stiffnesses}

Deformed unit-cells of a panel having narrow and wide gaps under in-plane shear strain $e_{12}$ and torsion strain $\chi_{12}$ are shown in Figure 11. The torsion behavior is for instance associated with floors supported on three sides and under out-of-plane loads. It is also involved in torsion deformation of simply supported floors in cases where the load is not symmetric.

When dealing with these generalized strains, all layers contribute to the stiffness of the panel. In the case of laterally glued CLT, global in-plane shearing or torsion of the panel occur and the continuous geometry ensures the stress transmission through the entire thickness. In the case of discontinuous CLT panels with gaps within each layer, the presence of free edges prevents the direct transmission of stresses between lamellas, leading to stress singularities (Figure 11a). As a consequence, the stresses increase due to the decrease of the net cross section (as also highlighted in [35] and [10]) and the global stiffness decreases. Such decrease of stiffness is also due to an additional compliance mechanism of rotation between the upper and lower lamellas that appears when the lateral edges are not glued. The reduction of in-plane stiffness becomes more significant in the case of large gaps, where all lamellas are submitted to bending in their plane due to their slenderness (Figure 11b).

Moosbrugger et al. [9] derived a simplified closed-form solution for predicting the in-plane shear stiffness of CLT panels having short gaps. According to this method, the stiffness of a glued panel is reduced by a "torsion-like" mechanism, dependent on the ratio $w / h$, that appears when the lamellas are not laterally glued to each other. This is in order to take into account the additional mechanisms of relative rotation under in-plane shear described above. The derivation of this simplified method is based on a CLT representative volume of a subelement (RVSE) composed by two lamellas and therefore assuming an infinite number of layers. Hence, according to this method, the reduction of in-plane shear stiffness is independent from the number of layers. Moreover, Moosbrugger et al. [9] considered the gaps to be short beams and their bending and shear compliance are taken into account. Hence, since increasing the gaps means increasing the span of beams represented by gaps, such a simplified approach may also predict the stiffness for large gaps within the panel. The closed-form solution returns directly the reduction $G / G^{*}$, where $G$ is the reduced stiffness of spaced CLT and $G^{*}$ is the in-plane shear stiffness of solid wood $\left(G_{L N}\right)$ that equals the stiffness of a continuous laterally glued CLT. This closed-form solution is compared in this section with the predicted $A_{33}$ and $D_{33}$ moduli normalized with the stiffnesses of a continuous CLT panel.

In Figure 12, the comparison between the experimental results of Brandner et al. [7] on the in-plane shear behavior of CLT with gaps and the predicted reduction of the modulus $A_{33}$ is presented. Both results from the plate homogenization procedure and from the closed-form solution [9] are shown. The experimental values are normalized with the reference experimental in-plane shear stiffness of a CLT having laterally glued lamellas, also given in [7]. Since in [7] the lamellas elastic properties were not provided, the in-plane shear stiffness is pre- 


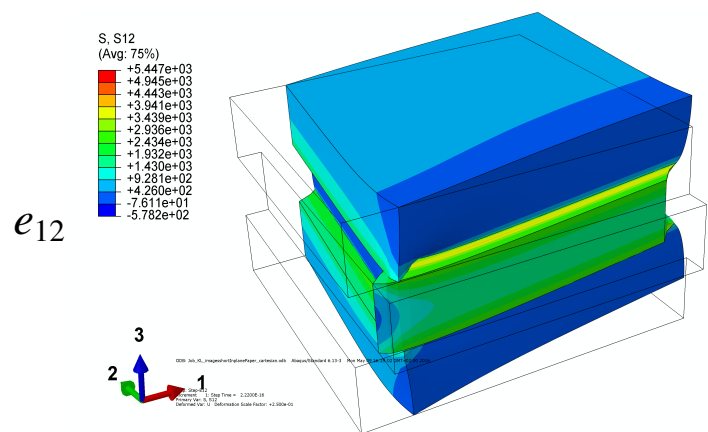

(a)

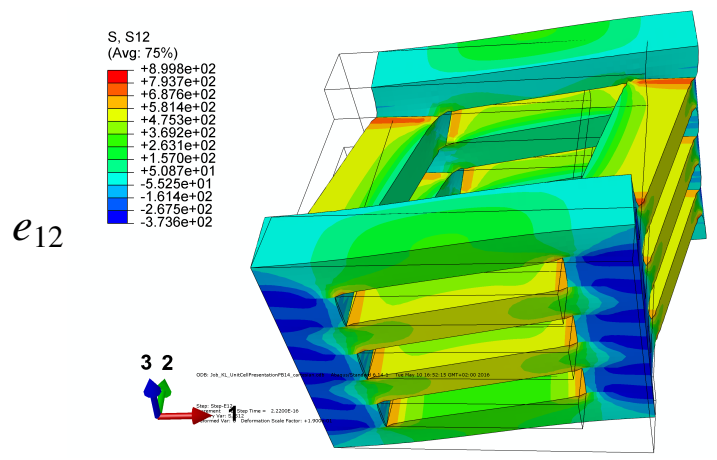

(b)

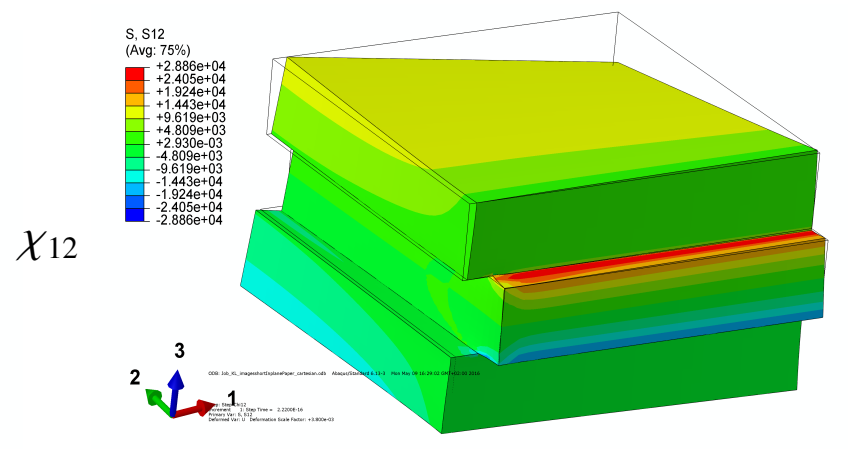

(c)

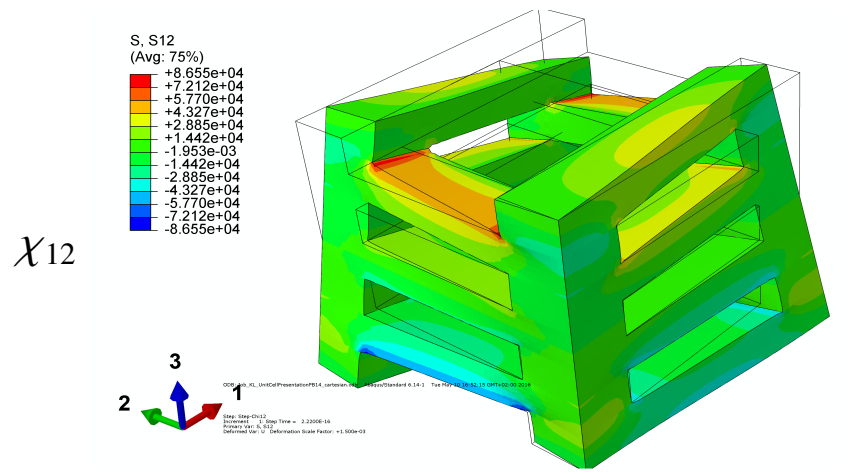

(d)

Figure 11: Deformed unit-cell of panels with gaps under in-plane shear at short (a) and large (b) gaps and under torsion at short (c) and large (d) gaps

dicted using the obtained timber properties showed in Table 3 and normalized with the corresponding stiffness as well.

When the lateral edges are not glued to each other, the mechanism of relative in-plane rotation of lamellas yields a sharp drop of in-plane shear stiffness that can be well predicted by the homogenization approach, while the closed-form approach underestimates the reduction of the stiffness by about $25 \%$.

As the study of Silly [33] has shown, the in-plane shear and torsional stiffnesses of CLT featuring gaps are influenced by the aspect ratio $w / h$. Therefore, Figure 13-16 plot the variation of normalized in-plane shear stiffness $A_{33}$ and torsional stiffness $D_{33}$ as a function of the gaps for CLT made up of lamellas having aspect ratio $w / h=3.33$ and $w / h=10$. Within each plot, the influence of the number of layers is highlighted as well.

Both the aspect ratio $w / h$ and the number of layers influence the in-plane shear and torsional stiffness of panels with gaps. Indeed, increasing the number of layers and the value of the ratio $w / h$ yields a lower drop of in-plane shear stiffnesses. The worst case of a 3-ply panel having lamellas with $w / h=3.33$ shows a $40 \%$ reduction of $A_{33}$ when the panel is not laterally glued and a $50 \%$ reduction for $6 \mathrm{~mm}$ gaps. For small gaps, the torsional stiffness $D_{33}$ shows higher reduction than the inplane shear stiffness and the same dependency on the number of layers. Interestingly, this trend is reversed when enlarging the gaps, where higher values of $D_{33}$ are found for 3-ply lay-ups and especially for high values of the ratio $w / h$.

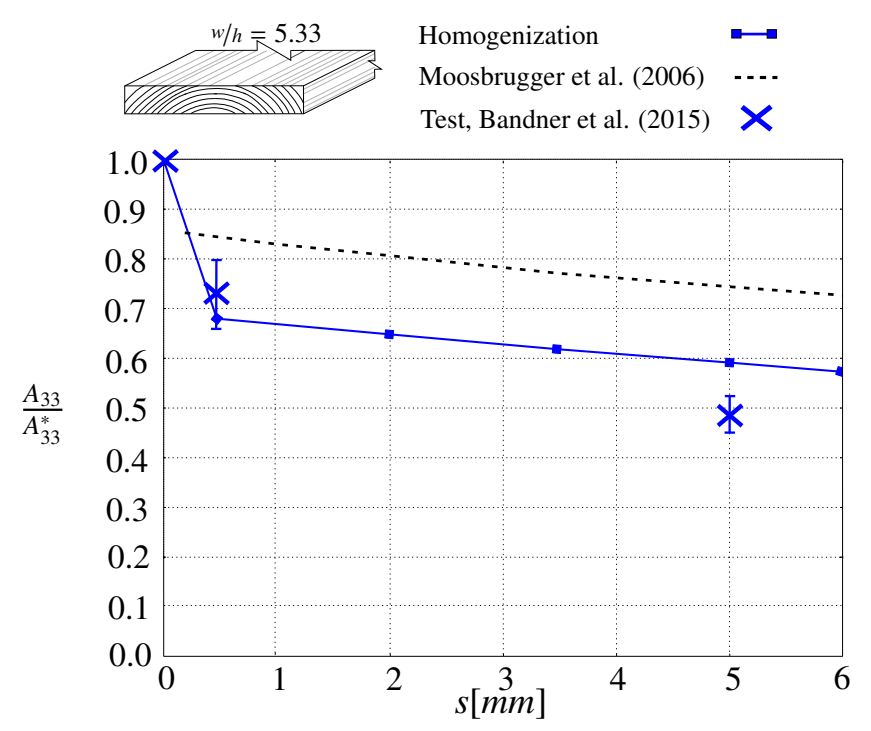

Figure 12: Comparison between test results and in-plane shear stiffness reduction $\frac{A_{33}}{A_{33}^{*}}$ for a 3-ply predicted with homogenization and closed-form solution 

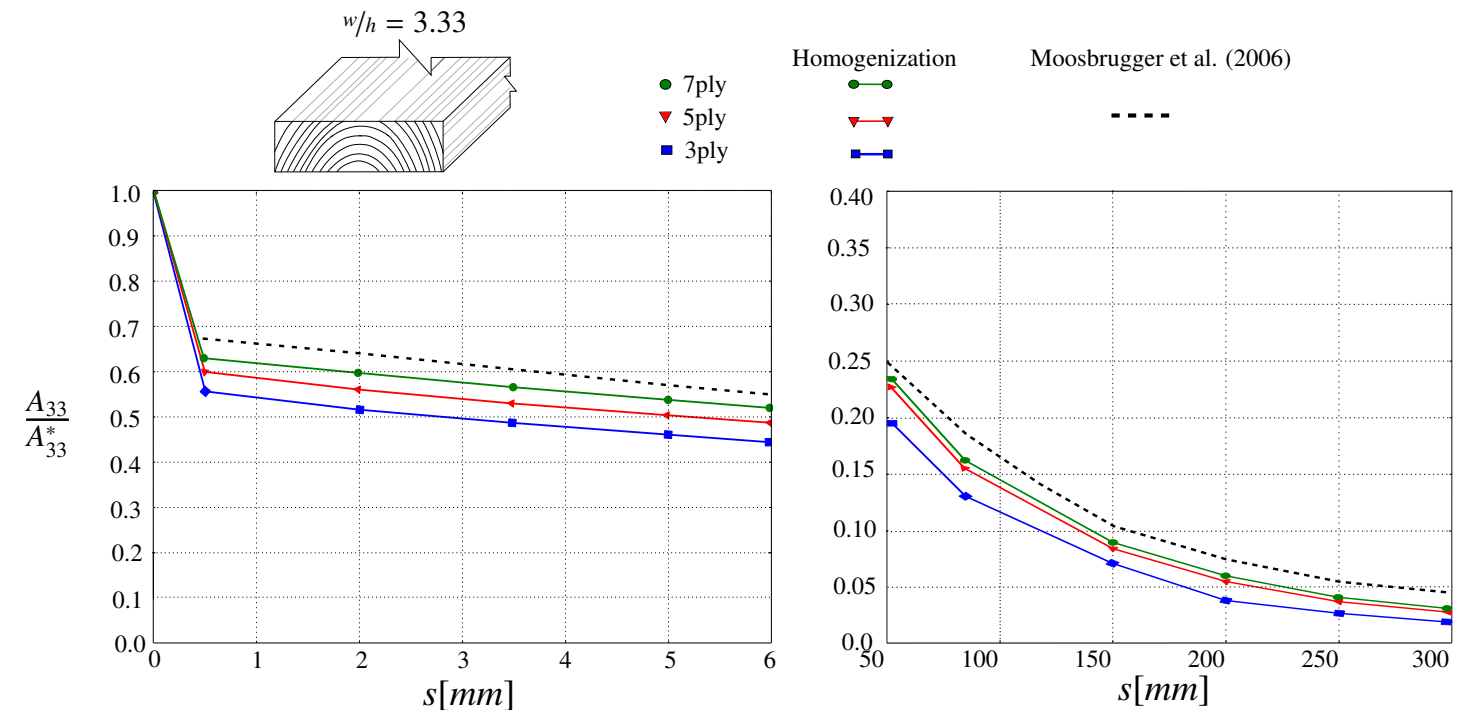

Figure 13: Variation of normalized in-plane shear stiffness $\frac{A_{33}}{A_{33}^{*}}$ for narrow (left) and wide (right) gaps for ratio $w / h=3.33$ of lamellas and for 3, 5 and 7 layers
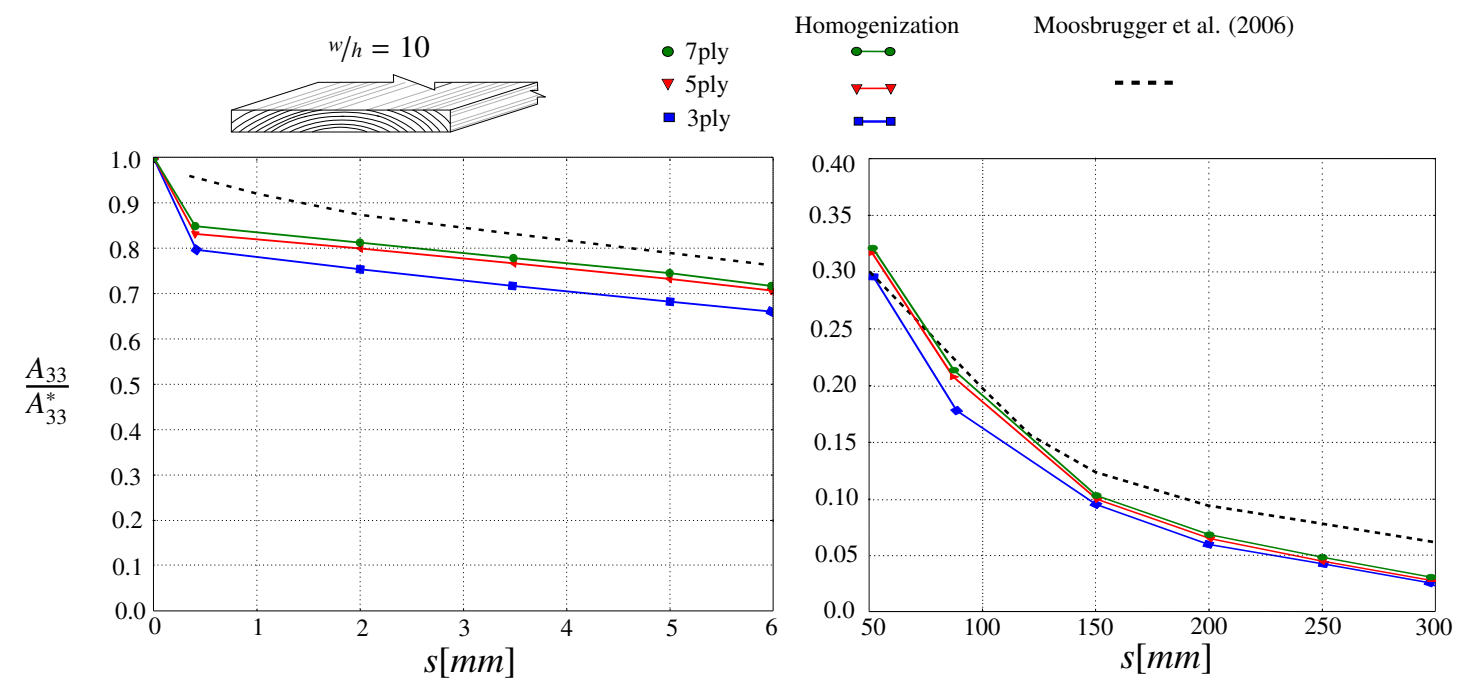

Figure 14: Variation of normalized in-plane shear stiffness $\frac{A_{33}}{A_{33}^{*}}$ for narrow (left) and wide (right) gaps for ratio $w / h=10$ of lamellas and for 3,5 and 7 layers
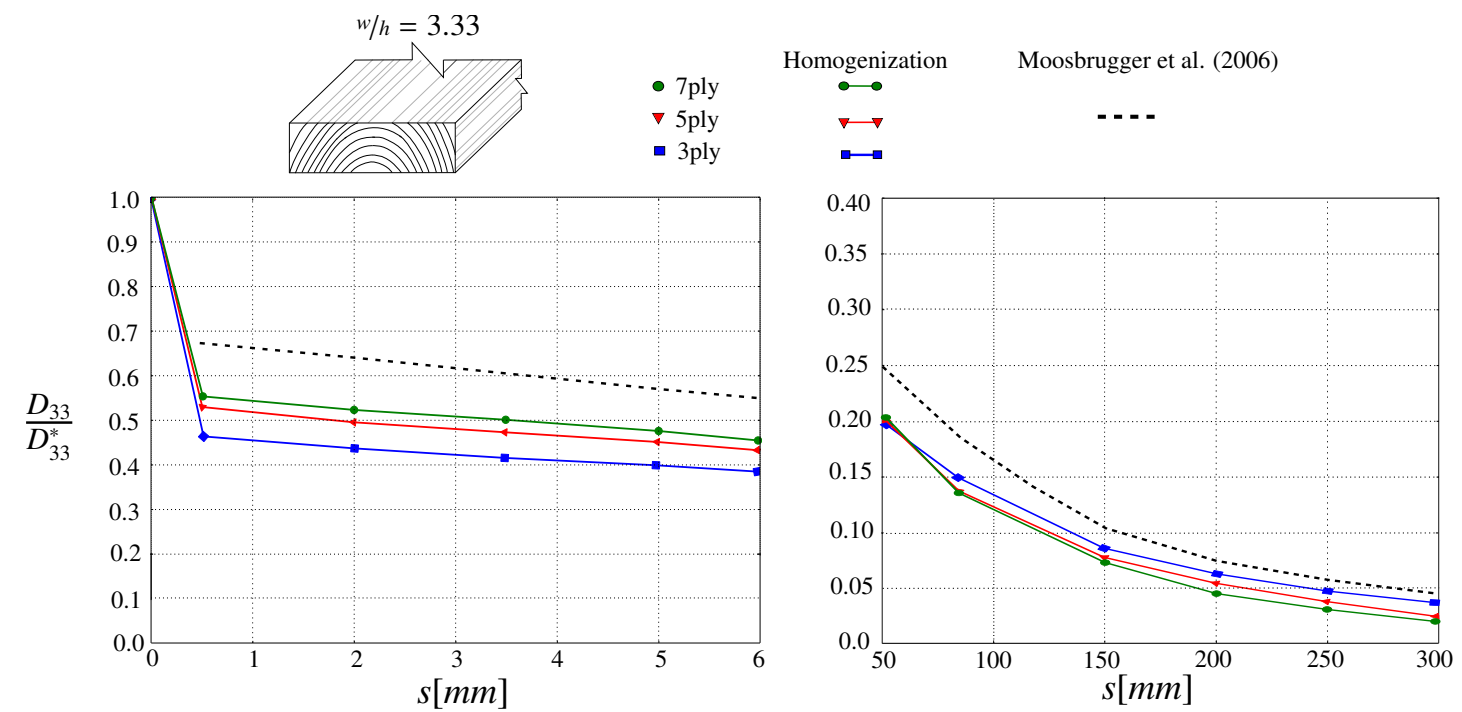

Figure 15: Variation of normalized torsional stiffness $\frac{D_{33}}{D_{33}^{*}}$ for narrow (left) and wide (right) gaps for ratio $w / h=3.33$ of lamellas and for 3,5 and 7 layers 

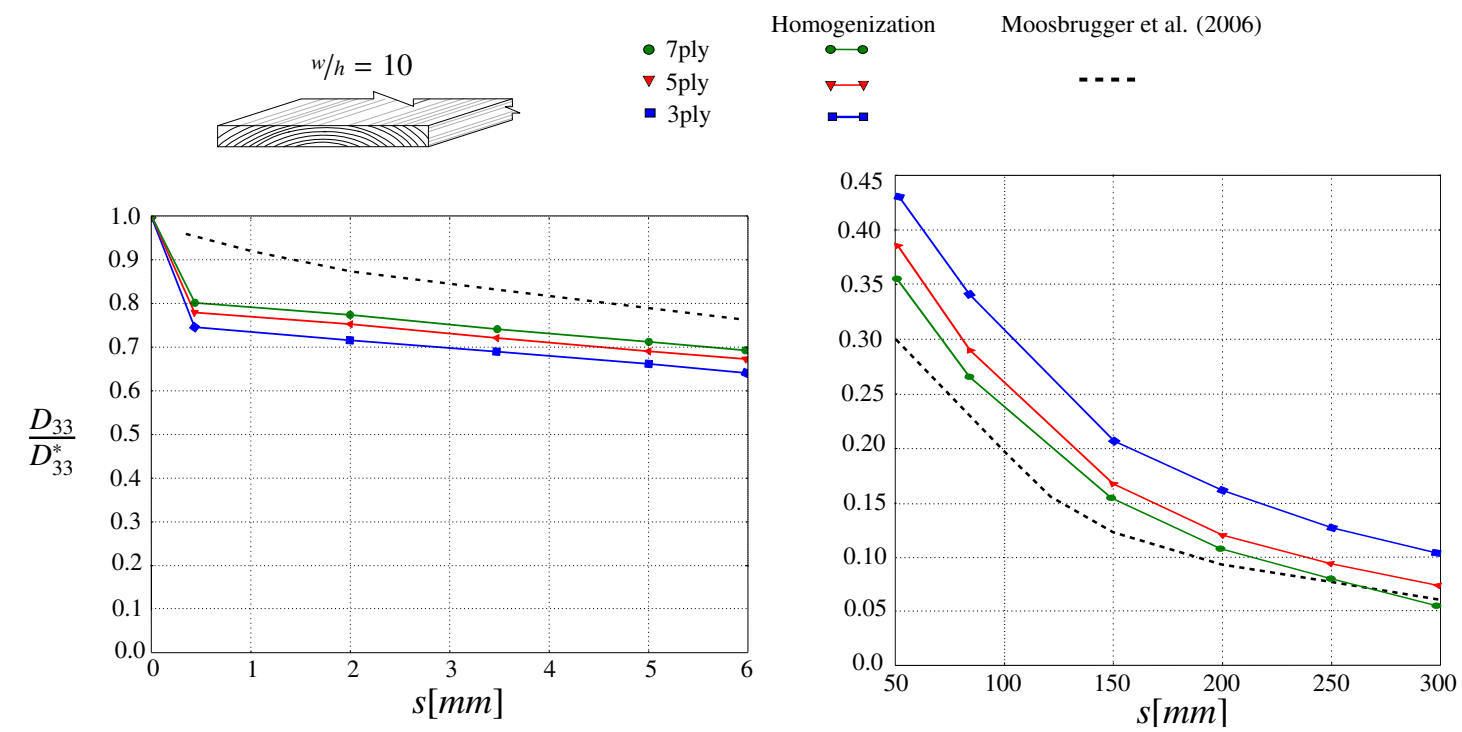

Figure 16: Variation of normalized torsional stiffness $\frac{D_{33}}{D_{33}^{*}}$ for narrow (left) and wide (right) gaps for ratio $w / h=10$ of lamellas and for 3,5 and 7 layers

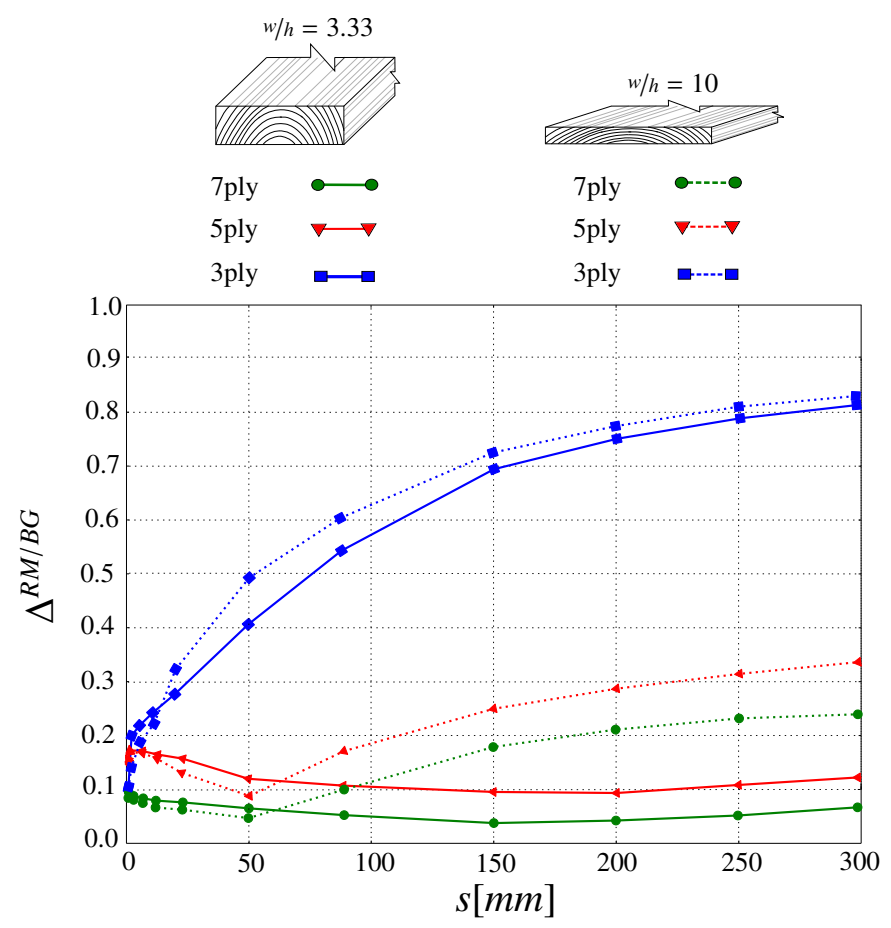

Figure 17: Distance between Reissner-Mindlin and Bending-Gradient plate models as a function of the width of the gaps and for different lay-ups

\subsection{Thick-plate homogenization}

The relative distance between the Reissner-Mindlin and the Bending-Gradient plate theories is plotted in Figure 17 as function of the size of gaps $s$ and for different lay-ups. As introduced in Section 3.1, if this distance is close to 1 the BendingGradient model is needed for a correct estimate of the shear force stiffness and stress distribution, while for distances close to 0 , a Reissner model may still be a reasonable approximation. The distance is not always small and is strongly influenced by the lay-up of the panel. For very large gaps all relative distances tend to $\frac{2 \sqrt{2}}{3}$ as predicted in [20], and it is the 3-ply configuration that goes more rapidly to this limit value. Additionally, thick lamellas $(w / h=3.33)$ yield lower values of the relative distance than thin lamellas $(w / h=10)$. However, except for the 3-ply layup, the relative distance remains moderate for the range of gaps interesting for practical applications. This allows the use of the more conventional Reissner-Mindlin plate theory.

Finally, the tested panels in 4-point bending were simply supported on two sides with the longitudinal layers aligned with the bending direction 1 , which is also called a cylindrical (or uni-axial) bending configuration. The relevant Reissner shear force stiffness $F_{11}$ is estimated from the Bending-Gradient theory as: $F_{11}=1 / h_{11111}=1 / h_{11}$ [32] and is investigated in this section.

Deformed unit-cells of CLT and panels with regular gaps under the cylindrical part of the generalized shear force $R_{111}$ are shown in Figure 18. In both cases, free edges lead to stress singularities close to interfaces. Further analysis revealed that an interaction between rolling-shear stresses $\left(\sigma_{13}\right)$ and perpendicular to grain stresses $\left(\sigma_{22}\right.$ and $\left.\sigma_{33}\right)$ is present. Such interaction have been proven to reduce the strength of lamellas having free narrow edges in recent experimental and numerical studies $[4,25]$. However, being a very localized phenomenon, the contribution of stress concentrations to the shear force stiffness is limited.

In this Section, the chosen simplified method for the prediction of the shear force compliance of spaced CLT is the shear analogy method derived by Kreuzinger [13] combined with the reduced properties using the wood volume fraction $\lambda$. In Figures 19 and 20 and Table 5, the normalized shear force stiffness $\frac{h_{11}^{*}}{h_{11}}$ predicted with the thick-plate homogenization and the volume fraction approach are compared to the shear force stiffness measured in 4-point bending tests. The shear force stiffness for short gaps within the panel predicted with the volume fraction is globally in agreement with experimental results and thick-plate homogenization (Figure 19). This is because when the gaps are 


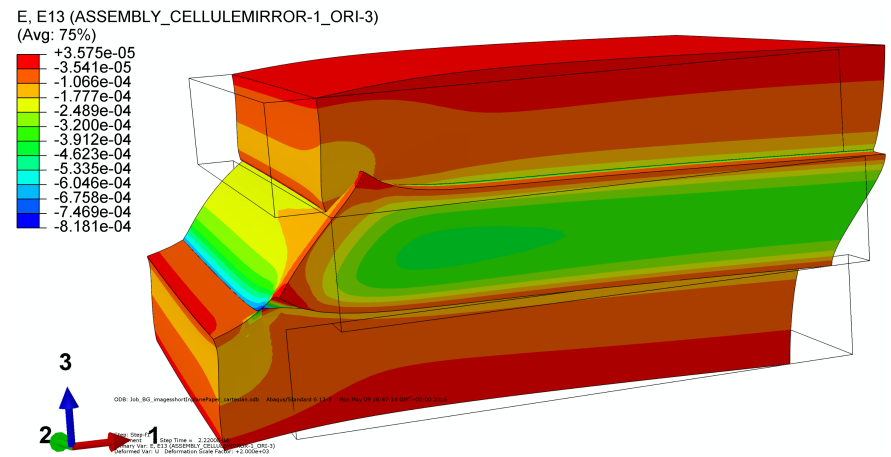

(a)

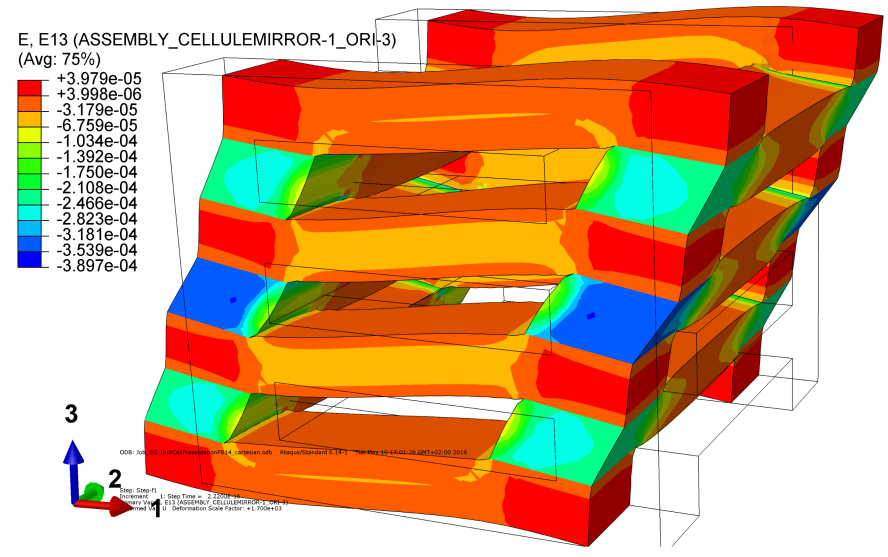

(b)

Figure 18: Deformed unit-cell under $R_{111}$ generalized shear force in the case of short gaps (a) $(\mathrm{w}=100, \mathrm{~h}=10, \mathrm{~s}=6)$ and large gaps $(\mathrm{b})(\mathrm{w}=100, \mathrm{~h}=30, \mathrm{~s}=150)$. The contour plot shows the transverse shear strain $\epsilon_{13}$.

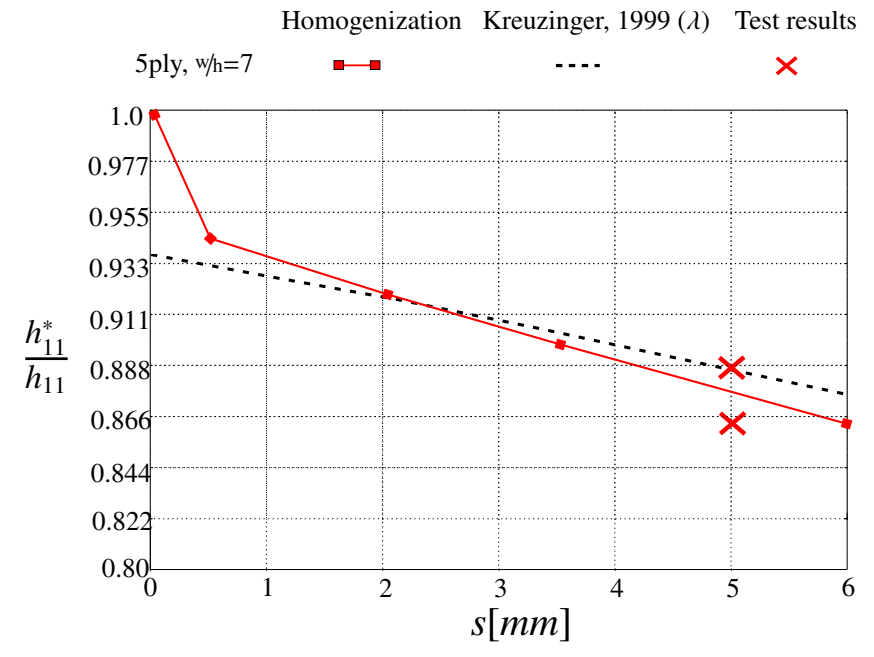

Figure 19: Variation of normalized shear force stiffness $\frac{h_{11}^{*}}{h_{11}}$ for short gaps and comparison with test results for the 5-ply

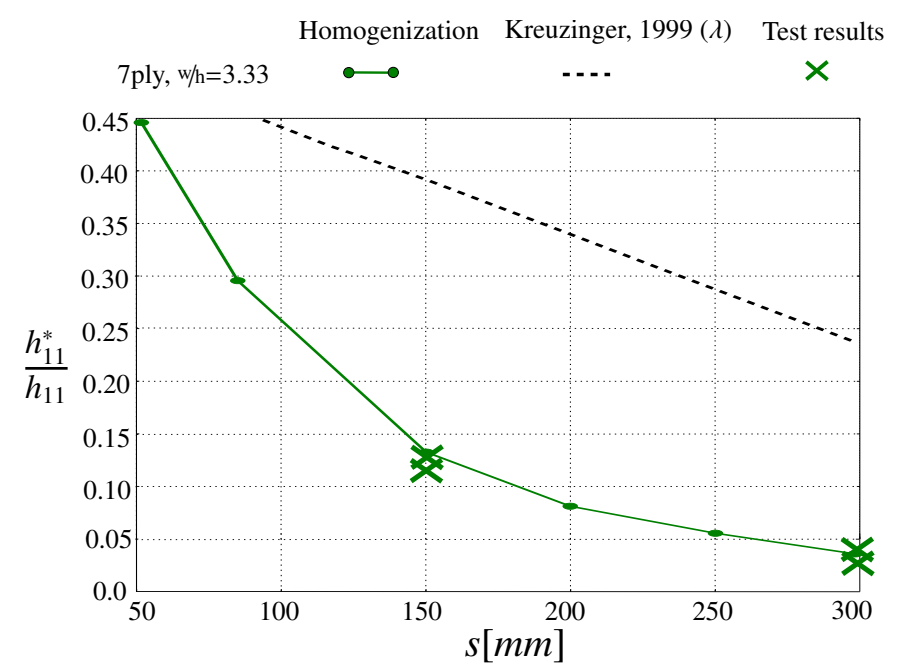

Figure 20: Variation of normalized shear force stiffness $\frac{h_{11}^{*}}{h_{11}}$ for large gaps and comparison with test results for the 7-ply

short the dominant contribution to the shear strain energy still derives from the rolling-shear of cross layers (see Figure 18a), exactly as in the case of laterally glued CLT. Figure 20 points out that the shear analogy method combined with the volume fraction strongly overestimates the shear force stiffness of panels having large gaps, while the thick-plate homogenization is in agreement with test results. This derives from the additional compliance mechanism caused by the longitudinal lamellas behaving as beams under simple bending, like a Verendeel beam (Figure 18b). Hence, this additional compliance mechanism cannot be taken into account in the simplified approach of volume fraction but only in the homogenization procedure.

In Figure 21 is plotted the variation of the shear force stiffness as function of increasing gaps and for two different aspect ratios $w / h$. As Figure 21a shows, already at short spaces there is a small drop of normalized shear force stiffness because of the free edges. This effect cannot be predicted by the volume fraction approach. 


\begin{tabular}{lccc}
\hline$h_{11}^{*} / h_{11}$ & Test result & Homogenization & Kreuzinger [13] \& $\lambda$ \\
\hline$s=5 \mathrm{~mm}(1)$ & 0.8574 & 0.8768 & 0.8874 \\
$s=5 \mathrm{~mm}(2)$ & 0.8872 & 0.8768 & 0.8874 \\
$s=150 \mathrm{~mm}(1)$ & 0.1272 & 0.1321 & 0.3925 \\
$s=150 \mathrm{~mm}(2)$ & 0.1251 & 0.1321 & 0.3925 \\
$s=300 \mathrm{~mm}(1)$ & 0.0392 & 0.0397 & 0.2453 \\
$s=300 \mathrm{~mm} \mathrm{(2)}$ & 0.0381 & 0.0397 & 0.2453 \\
\hline
\end{tabular}

Table 5: Variation of normalized shear force stiffness $\frac{h_{11}^{*}}{h_{11}}$ for small and wide gaps and comparison with test results
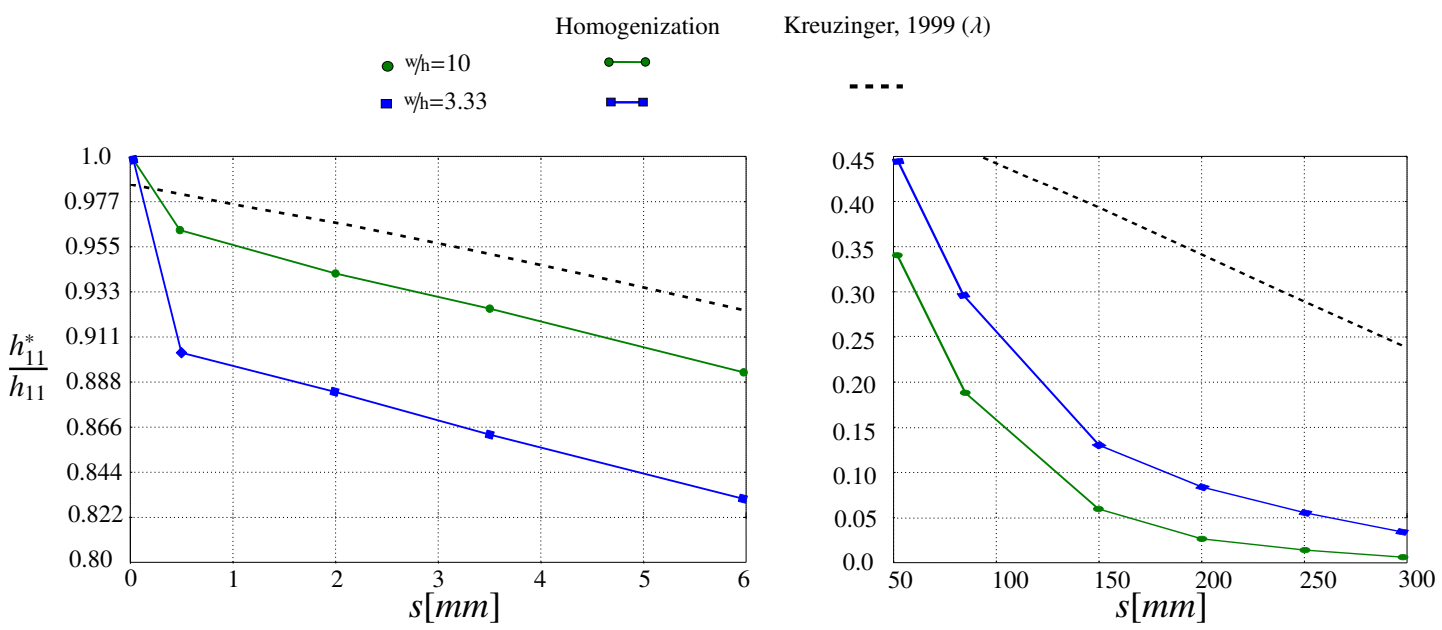

Figure 21: Variation of the normalized shear force compliance $\frac{h_{11}^{*}}{h_{11}}$ for a 7-ply at short (left) and large (right) gaps for different lamella ratio $w / h$

More precisely, in this case the number of free edges per unit length increases with the aspect ratio $w / h$. Hence, a higher drop of stiffness is observed with $w / h=3.33$ than with $w / h=10$ in Figure 21a. Enlarging the gaps means increasing the span of the longitudinal beams connected to cross lamellas. When such beams have small thickness, their slenderness further increases and their bending stiffness decreases. Therefore, as highlighted in Figure 21b and contrary to the small gaps case, panels having wide gaps and made of lamellas having high values of $w / h$ present lower shear force stiffness than panels with thicker lamellas.

\subsection{Influence of predicted stresses on the variation of failure modes}

The results presented in the previous sections concerned the variations of plate elastic moduli as a function of the size of gaps. The presence of wider gaps influences also the failure modes, as test results of Section 2 show. In addition to the global elastic energy stored in the unit-cell, the homogenization scheme predicts the 3D stress field inside the unit-cell. The actual stress field generated by the bending moment and the shear force can be rebuilt according to Equations (11) and (14). The predicted stress fields on a unit-cell have been successfully compared to a complete 3D FE simulation of the whole panel submitted to 4-point bending but not reported in this paper. When computing the stress field generated in the unit-cell by the failure load found experimentally, the stresses at failure $\sigma_{11}^{\max }$ and $\sigma_{13}^{\max }$ presented in Figure 22 can be found. Indeed the shear force $R_{111}$ is dominant close to supports and there is pure bending $M_{11}$ between loading forces.

Figure 22 shows that when the gaps between lateral lamellas are wider, the bending failure stress decreases while the rolling shear failure stress increases. The 4-point bending tests pointed out the transition between tensile parallel to grain failure of longitudinal layers to rolling shear failure of cross lamellas when enlarging the width of the gaps. Therefore, the predicted longitudinal and shear failure stresses may be compared to tensile parallel to grain and rolling-shear strength values of spruce. The mean tensile strength parallel to grain has been found to be approximately $30 \mathrm{MPa}$ by Stapel and van de Kuilen [36], Ranta-Maunus et al. [37] by means of tensile tests on thousands of spruce graded and ungraded lamellas having dimensions in the range of the lamellas of the panels tested in 4-point bending. Rolling-shear strength is considered to be independent from timber natural variability, as a recent comprehensive study pointed out [39], and mean rolling-shear strength of spruce has been found to be $1.6 \mathrm{MPa}$ by a previous study by the authors [27] with symmetric double-lap shear test and 1.8 MPa with two-plates shear test by Ehrart et al. [25].

Table 6 summarizes the predicted longitudinal and rollingshear stresses at failure for the three tested configurations in 4-point bending.

For the CLT ( $s=5 \mathrm{~mm}$ ), the predicted $\sigma_{11}^{\max }$ stress is close to the mean tensile strength of spruce, while the rolling-shear 


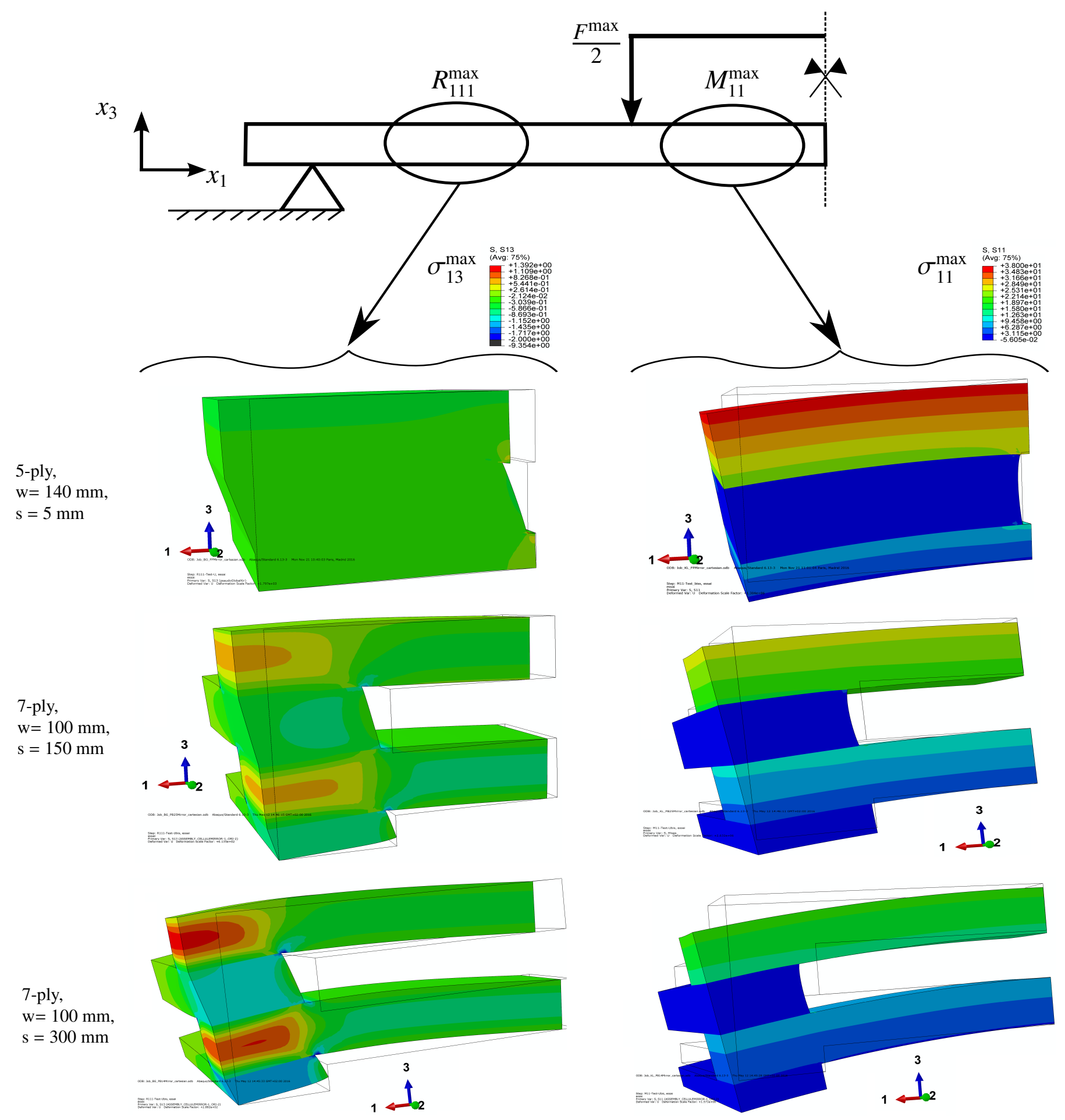

Figure 22: Variation of longitudinal $\sigma_{11}^{\max }$ (right) and rolling-shear $\sigma_{13}^{\max }$ (left) stresses under experimental failure bending moment $M_{11}^{\max }$ and shear force $R_{111}^{\max }$ for the three tested configurations 


\begin{tabular}{lccc}
\hline & Predicted $\sigma_{11}^{\max }[\mathrm{MPa}]$ & Predicted $\sigma_{13}^{\max }[\mathrm{MPa}]$ & Failure mode in tests \\
\hline$s=5 \mathrm{~mm}(1)$ & 37 & 0.52 & $\mathrm{TL}$ \\
$s=5 \mathrm{~mm}(2)$ & 39 & 0.55 & $\mathrm{TL}$ \\
$s=150 \mathrm{~mm}(1)$ & 26 & 0.97 & $\mathrm{TL}$ \\
$s=150 \mathrm{~mm}(2)$ & 24 & 0.88 & $\mathrm{TL}$ \\
$s=300 \mathrm{~mm}(1)$ & 18 & 1.37 & $\mathrm{RS}$ \\
$s=300 \mathrm{~mm}(2)$ & 18 & 1.42 & $\mathrm{RS}$ \\
\hline
\end{tabular}

Table 6: Variation of predicted longitudinal and rolling-shear stresses at failure of tested panels [MPa]. TL $=$ tensile longitudinal, $\mathrm{RS}=$ rolling-shear

failure stress is lower than the respective strength. This is in agreement with the experimental tensile failure of the bottom layer. The $s=150 \mathrm{~mm}$ configuration failed in tension in the bottom layer, even if the predicted $\sigma_{11}^{\max }$ decreases and $\sigma_{13}^{\max }$ increases, compared to the standard configuration. This is because of the presence of an isolated knot that led to tensile failure of both $s=150 \mathrm{~mm}$ panels (see Figure 4a). Indeed, standard CLT is characterized by a "system" effect that increases timber strength thanks to the glued surfaces between upper and lower lamellas, limiting the propagation of cracks due to local defects $[6,38]$. This effect is progressively reduced when enlarging the gaps, due to the increase in free unglued parts of the upper and lower lamellas, where the cracking of isolated knots is no longer mitigated. Finally, the rolling-shear stress predicted at failure of $s=300 \mathrm{~mm}$ panel in cross lamellas is very close to rolling-shear strength, while the predicted $\sigma_{11}^{\max }$ is lower than the tensile strength. This is in agreement with the experimental shear failure of cross lamellas of the $s=300 \mathrm{~mm}$ panel.

\section{Conclusions}

In the present paper, the elastic response of CLT panels and timber panels with regular gaps has been investigated by means of a thick plate homogenization scheme. The predicted results have been compared to experimental data and simplified closedform solutions. Both cases of narrow gaps allowed in standard panels and wide gaps in innovative timber products have been analyzed.

The experimental investigation pointed out the increasing influence of elastic and failure shear effects while enlarging the gaps between lamellas. This motivates the choice of the Bending-Gradient thick plate theory for a precise estimation of shear effects. The plate theory has been applied to the investigated configurations by means of a periodic homogenization scheme.

When increasing the gaps between lamellas, the reduction of the bending stiffness follows exactly the wood volume fraction within the panel. As consequence, the influence of narrow gaps is very small and the bending stiffness can be predicted, even for wide gaps, by the volume fraction approach. On the contrary, the reduction of in-plane shear and torsion stiffness does not follow the volume fraction and already at narrow gaps the reduction of stiffness is significant. The plate homogenization procedure returns a good agreement with in-plane shear test results from the literature. The reduction of the in-plane shear and torsional stiffness due to the gaps is influenced by the number of the layers and by the lamella's aspect ratio $w / h$. Increasing the number of layers as well as the aspect ratio $w / h$ leads to standard CLT and innovative panels with higher values of in-plane shear and torsional stiffness.

Regarding the out-of-plane shear behavior, the reduction of the shear force stiffness when enlarging the gaps cannot be predicted with the volume fraction approach due to an additional compliance mechanism related to simple bending of longitudinal lamellas. Therefore only the homogenization method can accurately predict the experimental results, while the volume fraction approach strongly overestimates the shear force stiffness. The presence of free edges in timber panels with gaps introduces an interaction between rolling-shear stress and traction perpendicular to the grain, leading to potential mixed failure modes in cross lamellas, as highlighted by the conducted 4-point bending tests. In standard CLT with unglued edges, the governing effect is rolling-shear of cross layers and therefore, for a fixed total thickness of the panel, the thicker the lamellas, the more shear compliant is the panel. On the contrary, concerning innovative panels with wide gaps, the simple bending of longitudinal layers due to shear force yields stiffer panels when the lamellas are thick.

The predicted values of longitudinal and rolling-shear failure stresses are in agreement with the experimental variation of failure modes and strength values from the literature, even if the natural variability of raw wood has not been taken into account and may be the subject of more accurate modeling.

In some cases, the existing closed-form approaches for predicting the mechanical behavior of both standard CLT and innovative panels are not appropriate. Nevertheless, the periodic homogenization presented in this paper requires the numerical solution of auxiliary problems by means of a FE software, that limits its implementation in practical applications. Hence, the derivation of closed-form solutions for predicting the elastic behavior of standard CLT and innovative panels is the object of on-going studies.

\section{Acknowledgments}

The authors gratefully acknowledge the producers Binderholz GMBH and Techniwood SAS for supplying the specimens for the experimental investigation. 


\section{References}

[1] European Committee of Standardization (CEN) 2015. EN 16351: Timber structures - Cross Laminated Timber - Requirements, Bruxelles (BEL)

[2] ANSI/APA PRG 320-2012 (2012). Standards for performance-rated cross-laminated timber, Tacoma (US)

[3] Brandner, R., Flatscher, D., Ringhofer, A., Schickhofer, G., Thiel, A 2016. Cross Laminated Timber (CLT): overview and development. European Journal of Wood and Wood products

[4] Hochreiner, G., Fussl, J., Eberhardsteiner, J. 2013. Cross Laminated Timber plates subjected to concentrated loading. Experimental identification of failure mechanisms. Strain 50 (1), 68-81

[5] Flores E.I.S., Saavedra, K., Hinojosa, J., Chandra, Y., Das, R. 2016 Multi-scale modeling of rolling shear failure in cross-laminated timber structures by homogenisation and cohesive zone models. International Journal of Solids and Structures 81, 219-232

[6] Franzoni, L., Lebée, A., Lyon, F., Foret, G. 2016. Influence of layers number and orientation on the elastic response and failure modes on CLT floors: modeling and parameter studies. European Journal of Wood and Wood Products

[7] Brandner, R., Dietsch, P., Droscher, J., Schulte-Wrede, M., Kreuzinger, H., Sieder, M., Schickhofer, G., Winter, S. 2015. Shear properties of Cross Laminated Timber (CLT) under in-plane load: test configuration and experimental study. Proceedings of the International Network on Timber Engineering Research, meeting forty-eight, Sibenik (CRO), 2427 August 2015

[8] Sebera, V., Muszynski, L., Tippner, J., Noyel, M., Pisaneschi, T., Sundberg, B. 2013. FE analysis of CLT panel subjected to torsion and verified by DIC. Materials and Structures 1 (9)

[9] Moosbrugger, T., Guggenberger, W., Bogensperger, T. 2007. Cross Laminated Timber segments under uniform shear, with and without openings. Proceedings of the 9th World Conference on Timber Engineering, Portland (USA)

[10] Bogensperger, T., Moosbrugger, T., Silly, G. 2010. Verification of CLT plates under loads in plane. Proceedings of the 11th World Conference on Timber Engineering, Trent (ITA)

[11] Hashin, Z. 1987. Analysis of orthogonally cracked laminates under tension. Journal of Applied Mechanics 54: 872-879

[12] Nairn, J. 2016. Mechanical properties of Cross Laminated Timber accounting for non-bonded edges and additional cracks. Proceedings of the 14th World Conference on Timber Engineering, Wien (AUT)

[13] Kreuzinger, H. 1999. Platten, Scheiben und Schalen. Ein Berechnungsmodell fur gangige Statikprogramme (in German). Bauen mit Holz, 1:34-39

[14] Blass, H. J., Gorlacher, R. 2000. Rolling shear in structural bonded timber elements. Proceedings of the International Conference on Wood and wood Fiber Composite

[15] Pagano, N., 1969. Exact solutions for composite laminates in cylindrical bending. Journal of Composite Materials 3 (3), 398-411

[16] Lebée, A., Sab, K. 2011. A bending-gradient model for thick plates part I: Theory. International Journal of Solids and Structures 48 (20), 2878-2888

[17] Sab, K., Lebée, A. 2015. Homogenization of heterogeneous thin and thick plates. Wiley-ISTE

[18] Lebée, A., Sab, K. 2011. A bending-gradient model for thick plates, part II: Closed-form solutions for cylindrical bending of laminates. International Journal of Solids and Structures 48 (20), 2889-2901

[19] Lebée, A., Sab, K., Oct. 2012. Homogenization of thick periodic plates: Application of the Bending-Gradient plate theory to a folded core sandwich panel. International Journal of Solids and Structures 49, 27782792

[20] Lebée, A., Sab, K., Oct. 2013. Homogenization of a space frame as a thick plate: Application of the Bending-Gradient theory to a beam lattice. Computers and Structures 70, 88-101

[21] Perret, O., Lebée, A., Douthe, C., Sab, K. 2016. The Bending-Gradient theory for the linear buckling of thick plates: Application to Cross Laminated Timber panels. International Journal of Solids and Structures $87,139-152$

[22] German Committee of Standardization (DIN) 2012. DIN 4074-1:201206: Strength classes for Norway spruce timber, Berlin (GER)
[23] European Committee of Standardization (CEN) 2009. EN 338: Timber structures - Strength classes, Bruxelles (BEL)

[24] Keunecke et al. (2008) Three-dimensional elastic behaviour of common yew and Norway spruce. Wood Science and Technology 42, 633647

[25] Ehrart et al. 2016. Rolling Shear Properties of some European Timber Species with Focus on Cross Laminated Timber (CLT):Test Configuration and Parameter Study. International Network on Timber Engineering Research, meeting forty-eight, Sibenik (CRO), 24-27 August 2015

[26] Zhou, Q., Gong, M., Chui, Y., Mohammad, M. 2014. Measurement of rolling shear modulus and strength of cross laminated timber fabricated with black spruce. Construction and Building Materials 79, 379-386

[27] Franzoni, L., Lebée, A., Lyon, F., Foret, G. 2015. Advanced modeling for design helping of heterogeneous CLT panels in bending. International Network on Timber Engineering Research, meeting forty-eight, Sibenik (CRO), 24-27 August 2015

[28] Franzoni, L., Lebée, A., Lyon, F., Foret, G. 2016. Bending behavior of regularly spaced CLT panels. In proceeding of the 14th World Conference on Timber Engineering, Vienna, (AUT) 22-25 August 2016

[29] Caillerie, D., 1984. Thin elastic and periodic plates. Mathematical Methods in the Applied Sciences 6 (2), 159 - 191

[30] Kohn, R., Vogelius, M. 1984 A new model for thin plates with rapidly varying thickness. International Journal of Solids and Structures 20 (4), 333-350

[31] Lebée, A., Sab, K., 2015. On the generalization of Reissner plate theory to laminatedplates, Part I: Theory. Journal of Elasticity

[32] Lebée, A., Sab, K., 2015. On the generalization of Reissner plate theory to laminatedplates, Part II: Comparison with the Bending-Gradient theory. Journal of Elasticity

[33] Silly, G. 2010. Numerische Studien Zur Drill-und Schubsteifigkeit von Brettsperrholz (BSP). (Numerical study on torsional stiffness of CLT plates) Diploma thesis, Institute for Timber Engineering and Wood Technology, TU Graz (in German)

[34] ABAQUS/Standard user's manual, version 6.13

[35] Brandner, R., Bogensperger, T., Schickhofer, G. 2013. In plane shear strength of cross laminated timber (CLT): test configuration, quantification and influencing parameters. Proceedings of the CIB-W18 meeting forty-six, Vancouver (CAN), 2013

[36] Stapel, P., van de Kuilen, J.W.G 2014. Influence of cross section and knot assessment on the strength of visually graded Norway spruce. European Journal of Wood and Wood Products, 72:213-227

[37] Ranta-Maunus, A., Denzler, J., Stapel, P. 2011. Strength of European Timber. Part 2: properties of spruce and pine tested in Gradewood project. VTT working papers 179, VTT Technical Research Center of Finland publishing

[38] Flaig, M., Blass, H.J. 2014. Bending strength of Cross Laminated Timber beams loaded in plane. Proceedings of the 13th World Conference on Timber Engineering, Quebec (CAN)

[39] Grandvuinet, T. Muszyński, T. 2016. Effects of knots and slope of grains on the rolling shear in dimensional timber used in CLT core layers. Proceedings of the 14th World Conference on Timber Engineering, Wien (AUT) 